\title{
Probing the cold and warm molecular gas in the Whirlpool Galaxy: Herschel SPIRE-FTS observations of the central region of M51 (NGC 5194)
}

M. R. P. Schirm, ${ }^{1}$ C. D. Wilson, ${ }^{1 \star}$ J. Kamenetzky, ${ }^{2,3}$ T. J. Parkin, ${ }^{1}$ J. Glenn, ${ }^{4}$ P. Maloney, ${ }^{4}$ N. Rangwala,${ }^{5}$ L. Spinoglio, ${ }^{6}$ M. Baes, ${ }^{7}$ A. Boselli, ${ }^{8}$ A. Cooray, ${ }^{9}$ I. De Looze, ${ }^{7,10}$ J. A. Fernández-Ontiveros, ${ }^{6}$ O. Ł. Karczewski ${ }^{11}$ and R. Wu ${ }^{12} \dagger$

${ }^{1}$ Department of Physics and Astronomy, McMaster University, Hamilton, ON L8S 4M1, Canada

${ }^{2}$ Steward Observatory, University of Arizona, 933 North Cherry Avenue, Tucson, AZ 85721, USA

${ }^{3}$ Westminster College, 1840 S 1300 E Salt Lake City, UT 84105, USA

${ }^{4}$ Center for Astrophysics and Space Astronomy, 389-UCB, University of Colorado, Boulder, CO 80303, USA

${ }^{5}$ Space Science and Astrobiology Division, NASA Ames Research Center, Moffet Field, CA 94035, USA

${ }^{6}$ Istituto di Astrofisica e Planetologia Spaziali, INAF-IAPS, Via Fosso del Cavaliere 100, I-00133 Roma, Italy

${ }^{7}$ Sterrenkundig Observatorium, Universiteit Gent, Krijgslaan 281 S9, B-9000 Gent, Belgium

${ }^{8}$ Aix Marseille Université, CNRS, LAM (Laboratoire d'Astrophysique de Marseille), UMR 7326, F-13388 Marseille, France

${ }^{9}$ Department of Physics and Astronomy, University of California, Irvine, CA 92697, USA

${ }^{10}$ Department of Physics and Astronomy, University College London, Gower Street, London WC1E 6BT, UK

${ }^{11}$ Laboratoire AIM, CEA/DSM - CNRS - Université Paris Diderot, IRFU/Service d'Astrophysique, CEA Saclay, F-91191 Gif-sur-Yvette, France

${ }^{12}$ Department of Astronomy, the University of Tokyo, Bunkyo-ku, Tokyo 113-0033, Japan

Accepted 2017 June 16. Received 2017 June 15; in original form 2016 May 11

\begin{abstract}
We present Herschel Spectral and Photometric Imaging Receiver (SPIRE)-Fourier Transform Spectrometer (FTS) intermediate-sampled mapping observations of the central $\sim 8 \mathrm{kpc}$ $(\sim 150 \mathrm{arcsec})$ of M51, with a spatial resolution of 40 arcsec. We detect four ${ }^{12} \mathrm{CO}$ transitions $(\mathrm{J}=4-3$ to $\mathrm{J}=7-6)$ and the $\left[\mathrm{C}_{\mathrm{I}}\right]{ }^{3} \mathrm{P}_{2}-{ }^{3} \mathrm{P}_{1}$ and ${ }^{3} \mathrm{P}_{1}-{ }^{3} \mathrm{P}_{0}$ transitions. We supplement these observations with ground-based observations of ${ }^{12} \mathrm{CO} \mathrm{J}=1-0$ to $\mathrm{J}=3-2$ and perform a two-component non-local thermodynamic equilibrium analysis. We find that the molecular gas in the nucleus and centre regions has a cool component $\left(T_{\text {kin }} \sim 10-20 \mathrm{~K}\right)$ with a moderate but poorly constrained density $\left(n\left(\mathrm{H}_{2}\right) \sim 10^{3}-10^{6} \mathrm{~cm}^{-3}\right)$, as well as significant molecular gas in a warmer $\left(T_{\text {kin }} \sim 300-3000 \mathrm{~K}\right)$, lower density $\left(n\left(\mathrm{H}_{2}\right) \sim 10^{1.6}-10^{2.5} \mathrm{~cm}^{-3}\right)$ component. We compare our $\mathrm{CO}$ line ratios and calculated densities along with ratios of $\mathrm{CO}$ to total infrared luminosity to a grid of photon-dominated region (PDR) models and find that the cold molecular gas likely resides in PDRs with a field strength of $G_{0} \sim 10^{2}$. The warm component likely requires an additional source of mechanical heating, from supernovae and stellar winds or possibly shocks produced in the strong spiral density wave. When compared to similar two-component models of other star-forming galaxies published as part of the Very Nearby Galaxies Survey (Arp 220, M82 and NGC 4038/39), M51 has the lowest density for the warm component, while having a warm gas mass fraction that is comparable to those of Arp 220 and M82, and significantly higher than that of NGC 4038/39.
\end{abstract}

Key words: ISM: molecules-galaxies: individual: NGC 5194-galaxies: ISM.

\section{INTRODUCTION}

M51 (NGC 5194) is a well-studied, relatively normal, nearby spiral galaxy. Its recent interaction with the nearby lenticular galaxy

*E-mail: wilsoncd@mcmaster.ca

$\dagger$ JSPS International Research Fellow.
NGC 5195 has led to triggered star formation throughout the galaxy (Nikola et al. 2001), and this interaction may be responsible for the prominent spiral arms of M51 (Zaritsky, Rix \& Rieke 1993; Dobbs et al. 2010). Rose \& Searle (1982) first suggested the presence of a non-stellar nuclear source of radiation at the centre of M51: it has been classified as a Seyfert type 2 (Seyfert-2) galaxy without a hidden broad-line region detected in polarized light (Tran 2001) and as a low-ionization nuclear emission-line region (LINER) galaxy 
(e.g. Satyapal, Sambruna \& Dudik 2004). The Seyfert-2 activity may have been triggered as a result of the interaction (Koulouridis 2014).

M51 is an excellent source in which to study both cold and warm molecular gas, due to its nearly face-on orientation, the prominence of its spiral arms and its recent interaction with NGC 5195. Observations of $\mathrm{H}_{2}$ rotational lines have found that the ratio of warm $(T=100-300 \mathrm{~K})$ to hot $(T=400-1000 \mathrm{~K})$ molecular gas varies across the system, which may suggest a varying excitation mechanism (Brunner et al. 2008). Roussel et al. (2007) found that the $\mathrm{H}_{2}$ is generally excited in photon-dominated regions (PDRs, also known as photodissociation regions). Recently, Parkin et al. (2013) modelled PDRs in M51 using various transitions of [O I], [C II] and $\left[\mathrm{N}_{\mathrm{II}}\right.$ ], along with the total infrared luminosity, and found that the far-ultraviolet (FUV) field strength necessary to reproduce PDRs in M51 varies in the range $G_{0} \sim 10^{1.5}-10^{4.0}$, with the highest values occurring in the nucleus.

The cold molecular gas has been studied predominantly through observations of ${ }^{12} \mathrm{CO}$ (hereafter $\mathrm{CO}$ ), and its isotopologues ${ }^{13} \mathrm{CO}$ and $\mathrm{C}^{18} \mathrm{O}$. M51 has been observed using ground-based single-dish telescopes in CO J = 1-0 (Scoville \& Young 1983; Garcia-Burillo, Guelin \& Cernicharo 1993; Nakai et al. 1994; Kramer et al. 2005; Koda et al. 2009), J = 2-1 (Garcia-Burillo, Guelin \& Cernicharo 1993; Kramer et al. 2005; Israel, Tilanus \& Baas 2006; Schuster et al. 2007; Leroy et al. 2009), J = 3-2 (Israel et al. 2006; Vlahakis et al. 2013) and $\mathbf{J}=4-3$ (Israel et al. 2006), and in ${ }^{13} \mathrm{CO} \mathrm{J}=1-$ 0 (Kramer et al. 2005), $\mathrm{J}=2-1$ (Kramer et al. 2005; Israel et al. 2006) and $\mathbf{J}=3-2$ (Israel et al. 2006). In addition, Israel et al. (2006) presented observations of $\left[\mathrm{C}_{\mathrm{I}}\right]$ in the ${ }^{3} \mathrm{P}_{1}-{ }^{3} \mathrm{P}_{0}$ (hereafter $\mathrm{J}=1-0$ ) transition at $492 \mathrm{GHz}$, which has also been proposed as a molecular gas tracer (e.g. see Papadopoulos, Thi \& Viti 2004; Offner et al. 2014).

Higher resolution interferometric observations of $\mathrm{CO} \mathrm{J}=2-1$, ${ }^{13} \mathrm{CO} \mathrm{J}=1-0$ and ${ }^{12} \mathrm{C}^{18} \mathrm{O} \mathrm{J}=1-0$ in M51 have been performed using the Owens Valley Radio Observatory (OVRO) by Schinnerer et al. (2010). These observations were limited to two regions within the spiral arms of M51. Using non-local thermodynamic equilibrium (non-LTE) excitation models with an escape probability formalism, they found that the temperature of the molecular gas in the observed giant molecular clouds (GMCs) is $T_{\text {kin }} \sim 20 \mathrm{~K}$. This temperature is similar to clouds in the Milky Way when observed at the same resolution $(\sim 180 \mathrm{pc})$.

More recently, M51 was observed at arcsecond resolution in $\mathrm{CO}$ $\mathrm{J}=1-0$ and ${ }^{13} \mathrm{CO} \mathrm{J}=1-0$ as part of the Plateau de Bure Interferometer (PdBI) Arcsecond Whirlpool Survey (PAWS; Hughes et al. 2013a,b; Meidt et al. 2013; Pety et al. 2013; Schinnerer et al. 2013; Colombo et al. 2014a,b). These observations were corrected for short spacings using single-dish observations. Colombo et al. (2014a) detect 1507 objects in CO $\mathrm{J}=1-0$, and find that the mass distribution, brightness and velocity dispersion of GMCs vary across the different environments in M51. Some of these differences seem to be dynamically driven (Meidt et al. 2013). Pety et al. (2013) detect extended $\mathrm{CO} \mathrm{J}=1-0$ emission that resides in a thick molecular disc with a scale height $\sim 200 \mathrm{pc}$, and accounts for $\sim 50$ per cent of the total CO J = 1-0 emission. Pety et al. (2013) suggest that this thick, extended disc could be the result of galactic fountains or chimneys due to the ongoing star formation.

In this paper, we present observations of M51 using the Spectral and Photometric Imaging Receiver (SPIRE; Griffin et al. 2010) Fourier Transform Spectrometer (FTS; Naylor et al. 2010) on board the ESA Herschel Space Observatory (Herschel; Pilbratt et al. 2010). The SPIRE-FTS is a low spatial and spectral resolution imaging spectrometer covering a spectral range from 194 to $671 \mu \mathrm{m}$ ( $\sim 450-1545 \mathrm{GHz})$. At the redshift of M51 $(z \sim 0.002)$, this spectral range includes a total of $10 \mathrm{CO}$ transitions $(\mathrm{J}=4-3$ to $\mathrm{J}=13-12)$, $10{ }^{13} \mathrm{CO}$ transitions $(\mathrm{J}=5-4$ to $\mathrm{J}=14-13)$ and two $\left[\mathrm{C}_{\mathrm{I}}\right]$ transitions $\left({ }^{3} \mathrm{P}_{1}-{ }^{3} \mathrm{P}_{0}\right.$ and ${ }^{3} \mathrm{P}_{2}-{ }^{3} \mathrm{P}_{1}$, hereafter $\mathrm{J}=1-0$ and $\mathrm{J}=2-1$, respectively) all of which trace molecular gas. The SPIRE-FTS [N II] $205 \mu \mathrm{m}$ data were previously published by Parkin et al. (2013); in this paper we present the detected $\mathrm{CO}$ and $\left[\mathrm{C}_{\mathrm{I}}\right]$ transitions for the first time. We adopt a distance to M51 of $9.9 \pm 0.7 \mathrm{Mpc}$ (Tikhonov, Galazutdinova \& Tikhonov 2009), based on observations of the tip of the red giant branch.

These observations were performed as part of the Very Nearby Galaxies Survey (VNGS; PI: C. D. Wilson) whose primary goal is to study the interstellar medium (ISM) of very nearby galaxies using both SPIRE and the Photoconductor Array Camera and Spectrometer (PACS; Poglitsch et al. 2010). From the sample of 13 galaxies in the VNGS, SPIRE-FTS CO data have been published for five: Arp 220 (Rangwala et al. 2011), M82 (Kamenetzky et al. 2012), NGC 1068 (Spinoglio et al. 2012), NGC 4038/39 (Schirm et al. 2014) and M83 (Wu et al. 2015). We present the observations and data reduction in Section 2. In Section 3 we present the non-LTE analysis of our detected $\mathrm{CO}$ and [ $\left.\mathrm{C}_{\mathrm{I}}\right]$ transitions, while in Section 4 we present models of PDRs in M51. We discuss the implications of the solutions of our non-LTE models and our PDR models in Section 5, along with a comparison of the results for M51 with previously studied galaxies within our sample.

\section{OBSERVATIONS}

\subsection{FTS data reduction}

M51 was observed using the SPIRE-FTS on OD 438 (2010 July $25)$ in intermediate-sampling mode, with 32 repetitions per jiggle position (Observation ID 1342201202). The observation is centred at $13^{\mathrm{h}} 29^{\mathrm{m}} 52^{\mathrm{s}} .71,+47^{\circ} 11^{\prime} 42^{\prime \prime}$. 60 , covering a region roughly $\sim 160 \times 180 \operatorname{arcsec}^{2}$ with a total integration time of $17603 \mathrm{~s}(\sim 5 \mathrm{~h})$. These data were reduced with the Herschel Interactive Processing Environment (HIPE) version 11.0 and SPIRE calibration 11.0. We used a modified version of the standard mapping pipeline (Swinyard et al. 2014), with the primary difference that we skip the map making step, instead saving each individual jiggle position as a level 1 spectrometer point source (SPS) product.

The standard mapping pipeline assumes either that the source is a point source or that the source is fully extended, filling the entire beam uniformly. As with many of the sources in the VNGS sample, M51 cannot be characterized as either a point source or a fully extended source relative to the FTS beam. In addition, the beam size and shape of the SPIRE-FTS varies with frequency, with the size varying from $\sim 17$ to $\sim 43$ arcsec. In previous works where we had fully Nyquist sampled maps of our sources (e.g. see Kamenetzky et al. 2012; Spinoglio et al. 2012; Schirm et al. 2014), we convolved our point-source calibrated integrated intensity maps using custom convolution kernels. The same technique cannot be used here as our map is not Nyquist sampled. Instead, we match the beam size across the entire spectrum using the recently developed semi-extended correction tool (SECT) in HIPE version 11.0 (Wu et al. 2013).

When a source is semi-extended, correcting for the FTS beam requires correcting for the source-beam coupling at every frequency. The SECT corrects for the source-beam coupling by assuming that the distribution of the emitting gas, whether it is [C $\mathrm{I}]$ - and CO-emitting molecular gas or $[\mathrm{N}$ II]-emitting ionized gas, follows the same spatial distribution as the selected reference image (Wu et al. 2013). It 
first calculates the source-beam coupling in the form of a forward coupling efficiency, $\eta_{\mathrm{f}}\left(v, \Omega_{\text {source }}\right)$, for each bolometer at a given jiggle position using derived FTS beam profiles and the normalized reference map. This source-beam coupling is frequency dependent, and so must be calculated at every frequency. It then multiplies the intensity at each frequency by this factor. The resulting data cube has an equivalent beam size and shape of a $40 \operatorname{arcsec}$ Gaussian beam. For our reference image, we opt to use the PACS $70 \mu \mathrm{m}$ image, which correlates strongly with star formation (Calzetti et al. 2010) and also has the advantage of having a significantly higher resolution than any of the FTS data (beam size $\sim 6$ arcsec). We expect that the molecular gas traced by $\mathrm{CO}$ and $\left[\mathrm{C}_{\mathrm{I}}\right]$ correlates well with the ongoing star formation in a relatively normal galaxy like M51. We perform this correction on the level 1 SPS products at each jiggle position using the SECT, with the correction varying between $\sim 0.5$ and $\sim 1.6$. The correction is dependent upon the frequency and the location of the bolometer.

We create a level 2 data cube for each set of detectors, the SPIRE Long Wavelength Spectrometer Array (SLW) and the SPIRE Short Wavelength Spectrometer Array (SSW), from the semicorrected level 1 products using the SPIREPROJECTION task. We chose a pixel size of 10 arcsec for both cubes. It is important to note that the pixel size will have no significant effect on our data cubes, provided we limit the pixel size such that only one detector is assigned to an individual pixel for each of the SLW and SSW level 2 data cubes. The resulting SLW and SSW data cubes contain 28 and 68 pixels with spectra, respectively. The complete semi-extended corrected FTS spectrum for the centre of M51 is shown in Fig. 1.

\subsubsection{Regions}

One of the aims of the VNGS is to investigate any regional variations in the ISM of the galaxies that we resolve. In the case of M51, we resolve the central $\sim 2 \operatorname{arcmin}$ at a beam size of 40 arcsec. We investigate the regional variations in the physical state and heating mechanisms of the molecular gas by assigning each pixel in our FTS map into one of four regions (see Fig. 2): the nucleus, centre, arm and interarm. We use the same region definitions as Parkin et al. (2013) to facilitate comparison with their results.

\subsubsection{Line fitting}

We wrote a custom line fitting routine in HIPE to fit all of the detected atomic and molecular transitions; a list of detected transitions is shown in Table 1. The intrinsic line profile of the FTS is a sinc function with a fixed full width at half maximum of $1.4305 \mathrm{GHz}$ in high-resolution mode, which corresponds to $280-450 \mathrm{~km} \mathrm{~s}^{-1}$ for the SSW, and 440-970 $\mathrm{km} \mathrm{s}^{-1}$ for the SLW in velocity space. ${ }^{1}$ The maximum measured line width from observations from the James Clerk Maxwell Telescope (JCMT) of CO J = 3-2 convolved to a beam size of $40 \operatorname{arcsec}$ (see Section 2.2.1) is only $\sim 50 \mathrm{~km} \mathrm{~s}^{-1}$ in the FTS field of view, less than the intrinsic line width of the instrument. Therefore, we do not resolve the line width in our observations.

For each pixel in our cubes, the routine fits each of the lines listed in Table 1 with a sinc function using a Levenberg-Marquardt fitter, keeping the width of the line fixed to $1.4305 \mathrm{GHz}$, with the amplitude and centroid varying. The surrounding $30 \mathrm{GHz}$ is fit with a quadratic at the same time in order to account for the continuum

${ }^{1}$ SPIRE Handbook. Available at: http://herschel.esac.esa.int/Docs/SPIRE /html/spire_om.html. Accessed 2015 July 14. emission. With the exception of the CO $\mathrm{J}=7-6$ and $\left[\mathrm{C}_{\mathrm{I}}\right] \mathrm{J}=2-1$ transitions, all of the lines listed in Table 1 are fit individually. In the case of the $\mathrm{CO} \mathrm{J}=7-6$ and $\left[\mathrm{C}_{\mathrm{I}}\right] \mathrm{J}=2-1$ transitions, both lines are fit concurrently, each with a sinc function, along with the continuum emission. We integrate the resulting sinc functions to calculate the total integrated intensity for each line, while the uncertainty is calculated from the uncertainty in the fitting parameters. The CO J = 10-9 line is detected only in the nucleus with a flux of $0.6 \pm 0.2 \mathrm{~K} \mathrm{~km} \mathrm{~s}^{-1}$. The calibration uncertainty of the SPIRE-FTS is 7 per cent, while we add a total of 10 per cent in quadrature to account for uncertainties in fitting the baseline, and uncertainties in the semi-extended source correction. The resulting maps for $\mathrm{CO}$ and $\left[\mathrm{C}_{\mathrm{I}}\right]$ are shown in Figs 3 and 4, respectively.

For each region, we perform an unweighted average for each $\mathrm{CO}$ and $\left[\mathrm{C}_{\mathrm{I}}\right]$ transition, including all pixels where all of the transitions are detected with a signal-to-noise ratio $(\mathrm{SNR})>1$. We opt for an unweighted average as the fluxes within an individual region are similar (Fig. 5). With the exception of the CO J $=8-7$ line, all of the transitions listed in Table 1 are detected with a SNR $>3$ for all pixels in the nucleus and centre regions. For the arm/interarm region, only a single pixel satisfies the same SNR $>3$. As such, we choose a SNR $>1$ in order to explore the parameter space in the arm and interarm regions of M51 (Fig. 5). Even with this low SNR cut, few pixels are included from the arm and interarm regions (see Fig. 5), while the included pixels all contain a portion of both regions within the 40 arcsec beam. Therefore, we combine the arm and interarm regions into a single arm/interarm region. Finally, we combine the pixels included in the nucleus, centre and arm/interarm regions into a single 'All' region.

\subsection{Ancillary data}

\subsubsection{Ground-based CO}

We supplemented our FTS observations of M51 using previously published $\mathrm{CO} \mathrm{J}=1-0$ to $\mathrm{J}=3-2$ maps from ground-based instruments. M51 was observed in CO J $=1-0$ and ${ }^{13} \mathrm{CO} \mathrm{J}=1-0$ using the Institut de Radioastronomie Millimétrique (IRAM) 30-m telescope as part of the PAWS program (Schinnerer et al. 2013). As we are interested only in the very large scales $(\sim 40 \mathrm{pc})$ in M51, we use the single-dish $\mathrm{CO} \mathrm{J}=1-0$ and ${ }^{13} \mathrm{CO} \mathrm{J}=1-0$ data cubes published in Pety et al. (2013), ${ }^{2}$ with a beam size of 22.5 arcsec. M51 was observed in CO J = 2-1 with the IRAM 30-m telescope as part of the HERA CO Line Extragalactic Survey ${ }^{3}$ (Schuster et al. 2007; Leroy et al. 2009). The publicly available data cube has been smoothed to 13 arcsec, the smallest beam size of all the CO maps used in this work. Finally, M51 was observed in CO J = 3-2 with 15 arcsec resolution by Vlahakis et al. (2013) using the HARP-B instrument on the JCMT. We use the data cube released as part of the JCMT Nearby Galaxies Legacy Survey ${ }^{4}$ (NGLS; Wilson et al. 2012).

All four of the ground-based CO transitions were reduced in the same manner using the STARLINK software package (Currie et al. 2008) and a similar method as for the $\mathrm{CO} J=3-2$ observations of NGC 4038/39 in Schirm et al. (2014). First, we convolved the data cubes to a 40 arcsec Gaussian beam using the GAUSMOоTH command. We then smoothed the cubes with a top-hat with a width

\footnotetext{
${ }^{2}$ Downloaded from http://www.mpia.de/PAWS/PAWS/Data.html

${ }^{3}$ http://www.mpia-hd.mpg.de/HERACLES/Overview.html

${ }^{4}$ http://www.physics.mcmaster.ca/ wilson/www_xfer/NGLS/
} 

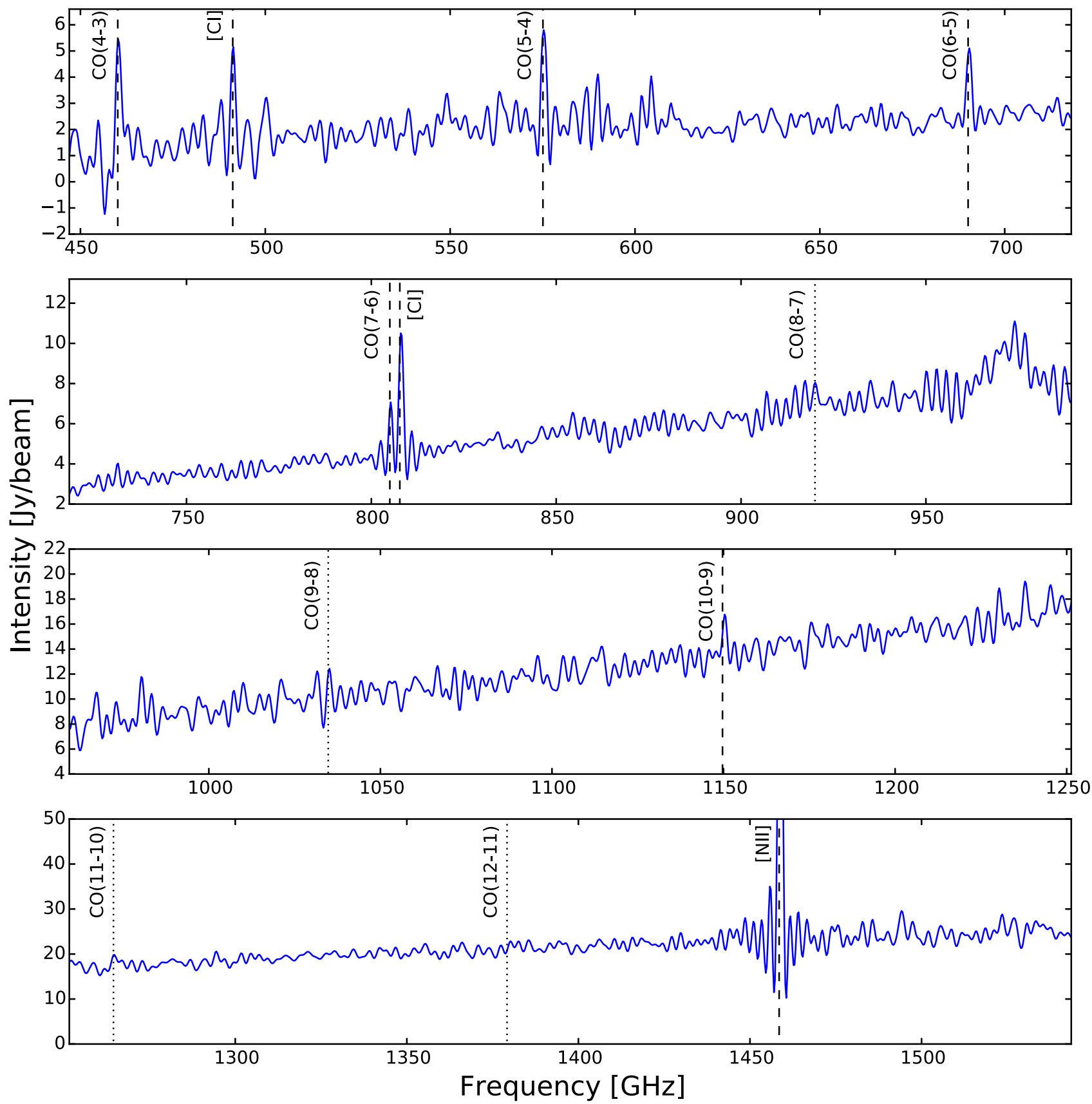

Figure 1. FTS spectrum for the nucleus of M51 in units of Jy beam ${ }^{-1}$. All of the detected atomic and molecular transitions are indicated by a dashed line, while undetected $\mathrm{CO}$ transitions are indicated by a dotted line at the expected location. This spectrum has been corrected for the semi-extended nature of the emission (see Section 2.1 for details). The peak of the [N II] line is at $\sim 100 \mathrm{Jy}_{\text {beam }}^{-1}$, beyond the scale of the plot. Note that CO J = 10-9 is detected only in the nucleus.

2.5 times the half-power beam-width (HPBW) of the $40 \operatorname{arcsec}$ beam and smoothed to a velocity width of $20 \mathrm{~km} \mathrm{~s}^{-1}$. Using the CLUMPFIND command, we identified regions of emission above $2 \sigma$ in our smoothed cube to create a mask that we then used to create moment 0,1 and 2 maps, corresponding the intensity-weighted integrated intensity, velocity and line-width maps, respectively, from our original, 40 arcsec HPBW, data cubes. We estimated the noise in our moment 0 maps using the emission free channels from our 40 arcsec HPBW data cubes. Finally, we regridded the ground-based data using the WCSALIGN task in STARLINK, using our CO J $=4-3$ integrated intensity map as a reference. The resulting spectral line energy distributions (SLEDs) for $\mathrm{CO}$ and $\left[\mathrm{C}_{\mathrm{I}}\right]$ are shown in Figs 5 (individual pixels) and 6 (region averages).

\subsubsection{Infrared data}

The total infrared luminosity, $L_{\mathrm{TIR}}$, can be combined with the $\mathrm{CO}$ line strengths to provide useful constraints on PDR models. We calculate the total infrared luminosity $\left(L_{\mathrm{TIR}}, \lambda=3-1100 \mu \mathrm{m}\right)$ using the following empirical relation from Galametz et al. (2013):

$L_{\mathrm{TIR}}=c_{24} v_{24} L_{24}+c_{70} v_{70} L_{70}+c_{160} v_{160} L_{160}$, 

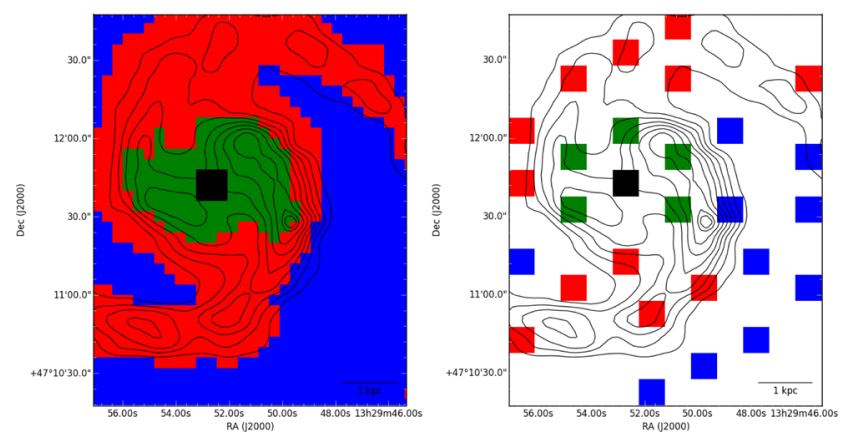

Figure 2. Left: schematic of M51 as defined in fig. 6 of Parkin et al. (2013) with CO J $=2-1$ contours overlaid. Right: the same regions of M51 defined for the pixels of our FTS maps with the same $\mathrm{CO} \mathrm{J}=2-1$ contours overlaid. In both figures, the colours correspond to the nucleus (black), centre (green), arm (red) and interarm(blue) regions of M51.

where the subscripts 24,70 and 160 correspond to the 24,70 and $160 \mu \mathrm{m}$ maps, respectively, while $c_{24}=2.133 \pm 0.095$, $c_{70}=0.681 \pm 0.028$ and $c_{160}=1.125 \pm 0.010$. We use the Spitzer Space Telescope Multiband Imaging Photometer for Spitzer (MIPS) $24 \mu \mathrm{m}$ map reprocessed by Bendo, Galliano \& Madden (2012), and the Herschel PACS 70 and $160 \mu \mathrm{m}$ maps from Mentuch Cooper et al. (2012). We beam-match and align all the maps using appropriate kernels from Aniano et al. (2011) before calculating the $L_{\mathrm{TIR}}$ map. We then beam-match and align the $L_{\mathrm{TIR}}$ map to our FTS observations using Gaussian kernels with the ІмSмоотн command to beam-match, and the IMREGRID command to regrid, in the Common Astronomy Software Package (CASA) version 4.2.1. The calculation of $L_{\mathrm{TIR}}$ using the 24,70 and $160 \mu \mathrm{m}$ photometric waveband provides a reliable estimate of $L_{\mathrm{TIR}}$ to within 25 per cent (Galametz et al. 2013).

\section{NON-LTE EXCITATION ANALYSIS}

We use the methods presented in Kamenetzky et al. (2014) to perform a non-LTE excitation analysis to determine the physical state of the molecular gas. Here, we present the highlights of the method used, along with any differences from the previous work. We use the non-LTE excitation code RADEX (van der Tak et al. 2007) along with a Bayesian likelihood code (Kamenetzky et al. 2014) to determine the kinetic temperature $\left(T_{\text {kin }}\right)$, molecular gas density $\left(n\left(\mathrm{H}_{2}\right)\right)$, area filling factor $\left(\Phi_{\mathrm{A}}\right)$ and $\mathrm{CO}$ column density per unit line width $\left(N_{\mathrm{CO}}\right)$. In this work, we also include the $[\mathrm{C} \mathrm{I}] / \mathrm{CO}$ column density ratio $\left(N_{\text {[C I }} / N_{\mathrm{CO}}\right)$ as one of the fitted parameters. The range of each parameter used in the models is given in Table 2. We compare our measured fluxes to the RADEX models to calculate the likelihood distribution for each of the physical parameters. The code determines the median and $1 \sigma$ range for each of the physical parameters from the marginalized likelihood distribution, along with the most probable 'best-fitting' ('4DMax') solution from the combined multidimensional likelihood distribution. By using Bayesian inference, we are able to include priors on the physical parameters based upon the physical characteristics of the observed region.

\subsection{Priors}

We introduce three priors into our modelling: the length along the line of sight, the total molecular gas mass and the optical depth. These are the same priors used in Schirm et al. (2014); however, the derivation of some of the physical parameters differs. The physical parameters used to calculate the priors are given in Table 3. Note that the line width, which is used to calculate the total column density $N_{\mathrm{CO}}$, is taken as the average from the CO $\mathrm{J}=3-2$ moment 2 map for all the pixels included in the regional averages.

The first prior is on the total length $(L)$ of the $\mathrm{CO}$ and [ $\left.\mathrm{C}_{\mathrm{I}}\right]$ emitting regions along the line of sight. This prior limits the column density, area filling factor and molecular gas density such that

$$
\frac{N_{\mathrm{CO}}}{\sqrt{\Phi_{\mathrm{A}}} x_{\mathrm{CO}} n\left(\mathrm{H}_{2}\right)} \leq L \text {. }
$$

As with all grand design spiral galaxies, the molecular gas in M51 resides predominantly in a disc. We derive our length prior based upon measurements of the scale height of this disc. Pety et al. (2013) calculated the scale height for their extended and compact components to be $\sim 190-250$ and $\sim 10-40$ pc, respectively (fig. 17 of their work). Their extended component corresponds to a warm, diffuse component, which we do not include in our models. (We discuss the implications of including a diffuse component in Section 5.1.3.) We use the scale height of their compact component and adopt a

Table 1. Line integrated intensity measurements.

\begin{tabular}{|c|c|c|c|c|c|c|c|}
\hline Species & Transition & $\begin{array}{l}\text { Rest frequency } \\
\quad(\mathrm{GHz})\end{array}$ & \multicolumn{4}{|c|}{$\begin{array}{l}\text { Average measured integrated intensity }{ }^{a} \\
\qquad\left(\mathrm{~K} \mathrm{~km} \mathrm{~s}^{-1}\right)\end{array}$} & $\begin{array}{c}\text { Calibration } \\
\text { uncertainty (per cent }\end{array}$ \\
\hline $\mathrm{CO}$ (ancillary) & $\begin{array}{l}\mathrm{J}=1-0 \\
\mathrm{~J}=2-1 \\
\mathrm{~J}=3-2\end{array}$ & $\begin{array}{l}115.27 \\
230.54 \\
345.80\end{array}$ & $\begin{array}{l}47.2 \pm 0.2 \\
37.8 \pm 0.2 \\
25.9 \pm 0.2\end{array}$ & $\begin{array}{l}41.2 \pm 0.1 \\
32.2 \pm 0.2 \\
20.9 \pm 0.2\end{array}$ & $\begin{array}{l}26.2 \pm 0.1 \\
20.0 \pm 0.2 \\
11.7 \pm 0.2\end{array}$ & $\begin{array}{l}33.1 \pm 0.1 \\
25.6 \pm 0.2 \\
16.0 \pm 0.2\end{array}$ & $\begin{array}{l}10.0^{b} \\
20.0^{c} \\
15.0^{d}\end{array}$ \\
\hline $\mathrm{CO}(\mathrm{FTS})$ & $\begin{array}{l}\mathrm{J}=4-3 \\
\mathrm{~J}=5-4 \\
\mathrm{~J}=6-5 \\
\mathrm{~J}=7-6 \\
\mathrm{~J}=8-7\end{array}$ & $\begin{array}{l}461.04 \\
576.27 \\
691.47 \\
806.65 \\
921.80\end{array}$ & $\begin{array}{c}12 \pm 1 \\
5.5 \pm 0.9 \\
2.2 \pm 0.1 \\
1.10 \pm 0.06 \\
<0.4 \pm 0.2\end{array}$ & $\begin{array}{c}9 \pm 1 \\
4.2 \pm 0.9 \\
1.5 \pm 0.4 \\
0.6 \pm 0.1 \\
<0.3 \pm 0.1\end{array}$ & $\begin{array}{l}<4.6 \pm 2.1 \\
<1.8 \pm 0.9 \\
<0.7 \pm 0.2 \\
0.28 \pm 0.07 \\
<0.3 \pm 0.1\end{array}$ & $\begin{aligned} 7 & \pm 2 \\
2.9 & \pm 0.9 \\
1.1 & \pm 0.3 \\
0.47 & \pm 0.08 \\
<0.3 & \pm 0.1\end{aligned}$ & $\begin{array}{l}12.2 \\
12.2 \\
12.2 \\
12.2 \\
12.2\end{array}$ \\
\hline${ }^{13} \mathrm{CO}$ (ancillary) & $\mathrm{J}=1-0$ & 110.20 & $5.87 \pm 0.06$ & $5.09 \pm 0.07$ & $2.92 \pm 0.06$ & $3.91 \pm 0.06$ & $10.0^{b}$ \\
\hline [C I] (FTS) & $\begin{array}{l}\mathrm{J}=1-0 \\
\mathrm{~J}=2-1\end{array}$ & $\begin{array}{l}492.16 \\
809.34\end{array}$ & $\begin{aligned} 8 & \pm 1 \\
3.08 & \pm 0.06\end{aligned}$ & $\begin{aligned} 6 & \pm 2 \\
1.9 & \pm 0.1\end{aligned}$ & $\begin{array}{l}<3.5 \pm 1.6 \\
0.85 \pm 0.08\end{array}$ & $\begin{array}{l}<4.6 \pm 1.6 \\
1.39 \pm 0.09\end{array}$ & $\begin{array}{l}12.2 \\
12.2\end{array}$ \\
\hline
\end{tabular}

Notes. ${ }^{a}$ Quoted uncertainties are measurement uncertainties only.

${ }^{b}$ Kramer, Moreno \& Greve (2008).

${ }^{c}$ Leroy et al. (2009).

${ }^{d}$ Vlahakis et al. (2013). 

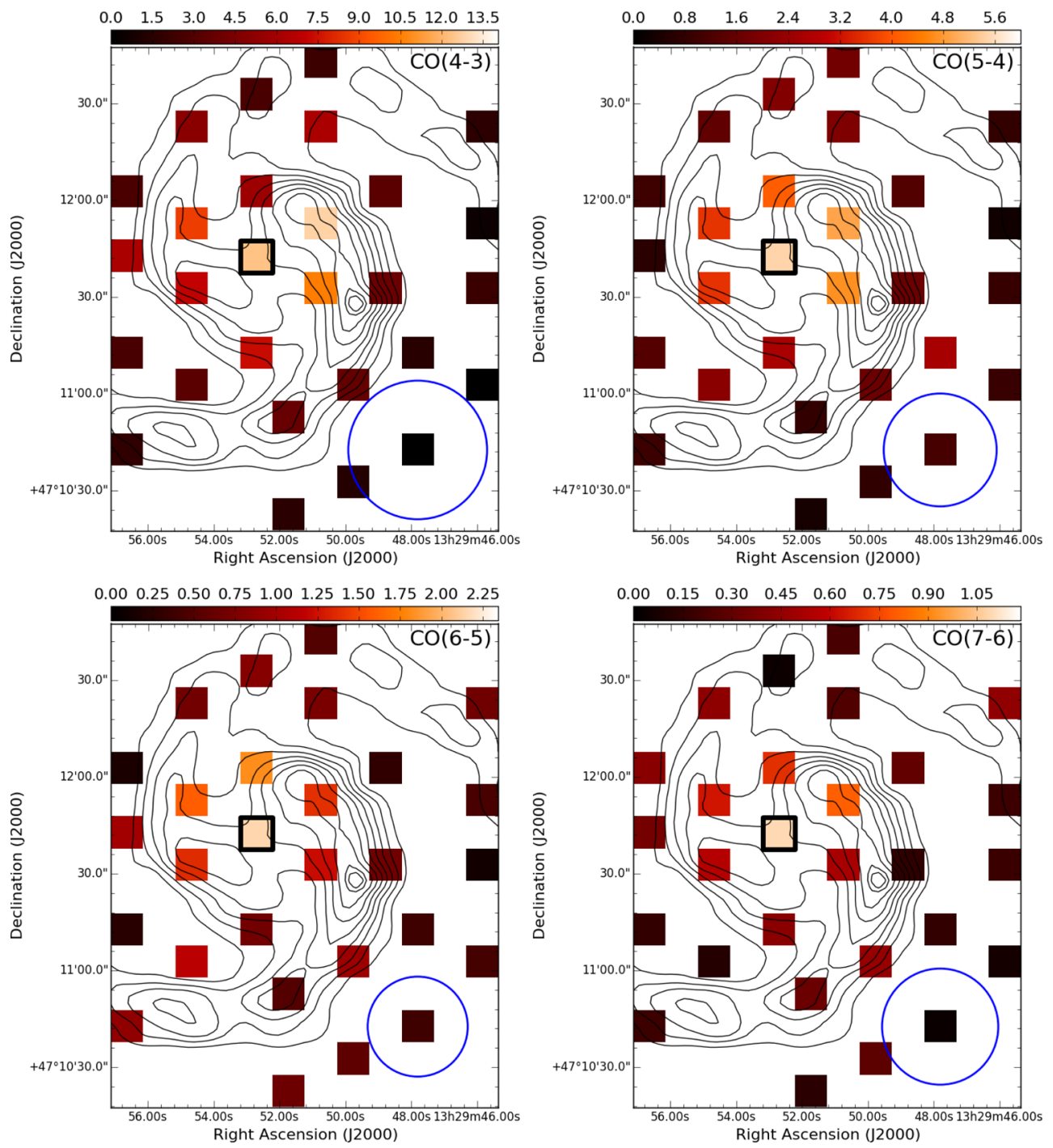

Figure 3. CO integrated intensity maps in units of $\mathrm{K} \mathrm{km} \mathrm{s}^{-1}$ from the Herschel SPIRE-FTS for the $\mathrm{J}=4-3$ (top left), $\mathrm{J}=5-4$ (top right), $\mathrm{J}=6-5$ (bottom left) and $\mathrm{J}=7-6$ (bottom right) transitions. The CO J = 2-1 contours from the IRAM 30-m telescope at a beam size of 13 arcsec are overlaid (Leroy et al. 2009). The CO integrated intensity maps shown here have been corrected for the semi-extended nature of the source (see Section 2.1). The native FTS beam size at the observed frequencies is shown as a blue circle in the bottom right-hand corner for each of the CO maps, while the centre of M51 is denoted by a black box. The semi-extended corrected maps have a 40 arcsec Gaussian beam, which is approximately the size of the CO J $=7-6$ beam.

length prior of $160 \mathrm{pc}$, a factor of 4 times the maximum scale height $(40 \mathrm{pc})$ derived for the compact component.

The second prior is on the total mass of molecular gas in a single beam. In previous publications (e.g. Rangwala et al. 2011; Kamenetzky et al. 2012; Schirm et al. 2014), we used the dynamical mass to limit the total molecular gas mass within a single beam. This assumption was sensible for systems contained entirely within a single FTS beam, such as Arp 220 (Rangwala et al. 2011). However, in the case of galaxies that span multiple beams, such as NGC 4038/39 (Schirm et al. 2014) or M51, it is more difficult to determine the dynamical mass per beam. Instead, we calculate an upper limit to the molecular gas mass for each pixel using the $\mathrm{CO} J=1-0$ map along with an $\alpha_{\mathrm{CO}}$ value of $9 \mathrm{M}_{\odot} \mathrm{pc}^{-2}\left(\mathrm{~K} \mathrm{~km} \mathrm{~s}^{-1}\right)^{-1}$. This value for the conversion factor corresponds to the largest value for $\alpha_{\mathrm{CO}}$ seen in the Milky Way and other Local Group galaxies $\left(\alpha_{\mathrm{CO}} \sim 3-\right.$ $9 \mathrm{M}_{\odot} \odot \mathrm{pc}^{-2}\left(\mathrm{~K} \mathrm{~km} \mathrm{~s}^{-1}\right)^{-1}$; Leroy et al. 2011). The mass prior for each region is the average from all the pixels included in the region average.

The mass prior places a limit on the beam-averaged column density $\left(\left\langle N_{\mathrm{CO}}\right\rangle=N_{\mathrm{CO}} \Phi_{\mathrm{A}}\right)$ :

$N_{\mathrm{CO}} \Phi_{\mathrm{A}}<\frac{M_{\mathrm{mol}} x_{\mathrm{CO}}}{\mu m_{\mathrm{H}_{2}} A_{\mathrm{CO}}}$,

where $M_{\mathrm{mol}}$ is our derived mass from the $\mathrm{CO} \mathrm{J}=1-0$ map, $x_{\mathrm{CO}}$ is the $\mathrm{CO}$ abundance relative to $\mathrm{H}_{2}, \mu$ is the mean molecular weight, $m_{\mathrm{H}_{2}}$ is the mass of the $\mathrm{H}_{2}$ molecule and $A_{\mathrm{CO}}$ is the area of the $\mathrm{CO}$ emitting region, which is the area covered by one beam at the distance of M51.

The third prior limits the optical depth of each line such that $-0.9<\tau<100$. An optical depth $<0$ is indicative of a maser, and we do not expect $\mathrm{CO}$ or $\left[\mathrm{C}_{\mathrm{I}}\right]$ masers to contribute appreciably to the emission on the observed size scales (the limit of -0.9 allows for 

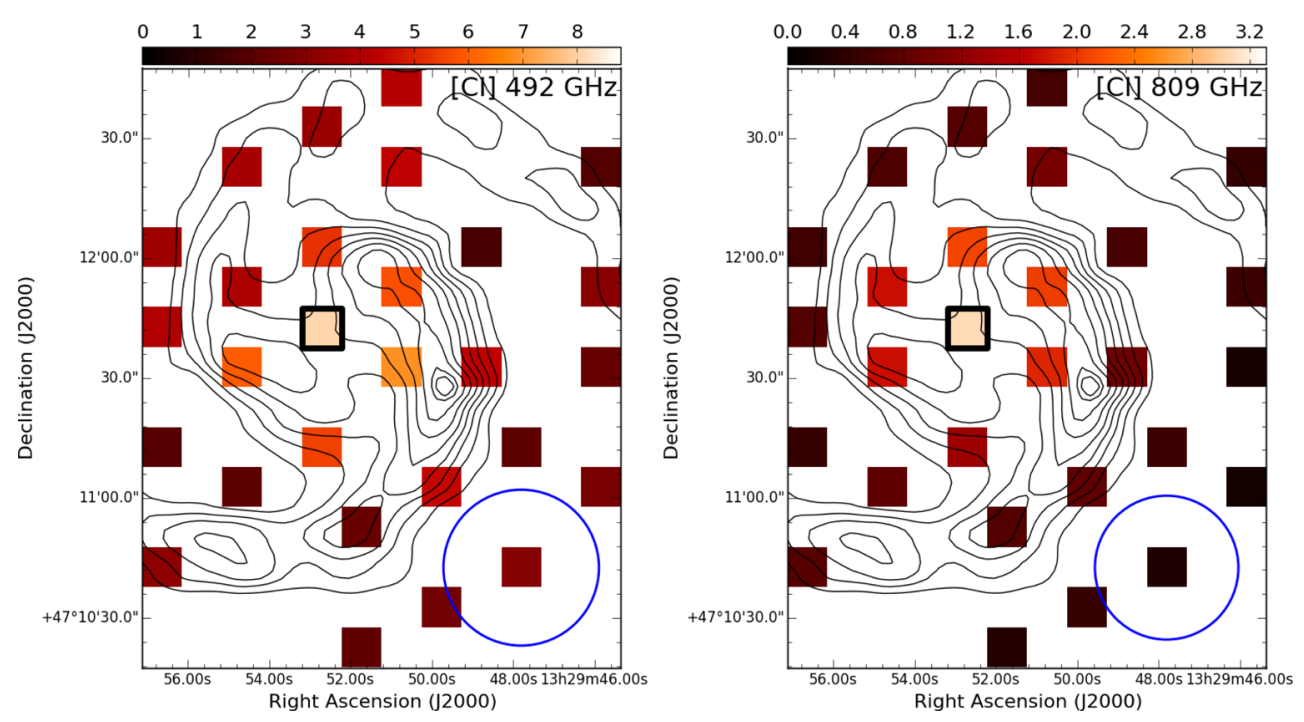

Figure 4. [C $\mathrm{I}]$ integrated intensity maps in units of $\mathrm{K} \mathrm{km} \mathrm{s}^{-1}$ from the Herschel SPIRE-FTS observations of M51 for the ${ }^{3} \mathrm{P}_{1}-{ }^{3} \mathrm{P}_{0}$ transition at $492 \mathrm{GHz}$ (left) and the ${ }^{3} \mathrm{P}_{2}-{ }^{3} \mathrm{P}_{1}$ transition at $809 \mathrm{GHz}$ (right) transitions. For more details see Fig. 3.

computational error). Furthermore, the line intensities calculated by RADEX become more uncertain the further the optical depth drops below 0 . In addition, van der Tak et al. (2007) suggest limiting the optical depth to an upper limit of 100, as the one-zone approximation implied by its escape probability formalism breaks down at optical depths greater than $\tau>100$.

\subsection{Non-LTE excitation modelling}

$\mathrm{CO},\left[\mathrm{C}_{\mathrm{I}}\right]$ and ${ }^{13} \mathrm{CO}$ are all tracers of molecular gas; all three species are excited via collisions with $\mathrm{H}_{2}$. In the classic slab-geometry model of a PDR by Tielens \& Hollenbach (1985), [C I] arises from the surfaces of molecular clouds, while $\mathrm{CO}$ does not begin to form until deeper into the cloud. In this model, some of the molecular gas is 'CO dark' (Wolfire, Hollenbach \& McKee 2010). However, there is strong evidence that $[\mathrm{C} \mathrm{I}]$ and $\mathrm{CO}$ are cospatial (Papadopoulos et al. 2004), as supported by observations of the Orion molecular cloud (Plume et al. 1999; Ikeda et al. 2002; Shimajiri et al. 2013) and more recent simulations of molecular clouds (e.g. Offner et al. 2014; Gaches et al. 2015). Furthermore, [C I] may be less sensitive to temperature than CO (Offner et al. 2014), and so may help constrain the density.

We fit a two-component model to our measured $\mathrm{CO}$ and $[\mathrm{C} \mathrm{I}]$ intensities in the nucleus, centre and arm/interarm regions of M51. (A single-component model fit, which does not produce a physically realistic solution, is discussed in Appendix A.) We include the $\mathrm{CO}$ transitions from $\mathrm{J}=1-0$ to $\mathrm{J}=7-6$, while leaving the $\mathrm{J}=8-7$ transition as an upper limit. The total uncertainty used is the line fitting and calibration uncertainties added in quadrature. The molecular gas in M51 is unlikely to populate two distinct components in terms of the physical state of the gas, so our two-component fit will represent an average of the state of all the molecular gas within the three distinct regions. We are therefore investigating the bulk properties of the molecular gas in the three regions. (For an extensive discussion of one- and two-component modelling, see Kamenetzky et al. 2014.)

Our two-component fit to the molecular gas consists of a 'cold' component that can dominate the lower J CO transitions, and a 'warm' component that dominates the upper J CO transitions. We include the $\left[\mathrm{C}_{\mathrm{I}}\right]$ in the cold component model only. Our initial tests fitting both components simultaneously while constraining the temperature of the cold component to be $<100 \mathrm{~K}$ resulted in a bimodal temperature distribution, with high probabilities at the upper $(100 \mathrm{~K})$ and lower $(10 \mathrm{~K})$ ends of the range and a minimum probability around $30 \mathrm{~K}$. Therefore, to isolate better the cold component from the warmer component, we reran the fits while constraining the temperature of the cold component to be $<10^{1.5} \mathrm{~K}$. The derived physical parameters are given in Table 4; we give both the median value for each parameter and the 'best-fitting' value, which is the set of values in the multidimensional parameter space with the highest probability. The resulting measured and calculated SLEDs are shown in Fig. 6, while the calculated optical depths are shown in Fig. 7. The $1 \sigma$ ranges are shown in Fig. 8 and an example of the probability distributions for four of the fit parameters is given in Appendix B.

\section{PHOTON-DOMINATED REGIONS}

PDRs are regions of molecular gas illuminated by FUV radiation $\left(6.20<E_{\text {phot }}<13.6 \mathrm{eV}\right.$; Tielens \& Hollenbach 1985). While FUV photons are typically not the right energy to dissociate molecular hydrogen, this radiation can have a significant effect on the chemistry and heating of the illuminated region. Indeed, the FUV radiation will liberate electrons from dust grains through the photoelectric effect, which in turn will heat the molecular gas through collisions.

Using a PDR model grid (Hollenbach et al. 2012; Wolfire, private communication), we model the ratio of $\mathrm{CO}$ transitions (e.g. $\mathrm{CO} \mathrm{J}=3-2 / \mathrm{J}=2-1$, etc.) for the nucleus, centre and arm/interarm regions of M51, along with the combination of all three regions. The model uses the molecular gas density $\left(n\left(\mathrm{H}_{2}\right)\right)$ and FUV field strength $\left(G_{0}\right)$ in units of the Habing field (FUV flux $\left.=1.3 \times 10^{-4} G_{0} \mathrm{erg} \mathrm{cm}^{-2} \mathrm{~s}^{-1} \mathrm{sr}^{-1}\right)$. The model grid spans a large range of density $\left(n\left(\mathrm{H}_{2}\right)=10^{1.0}-10^{7.0} \mathrm{~cm}^{-3}\right)$ and FUV field strengths $\left(G_{0}=10^{-0.5}-10^{6.5}\right)$ to calculate the CO fluxes for the transitions from $\mathrm{J}=1-0$ up to $\mathrm{J}=29-28$. We show the $\mathrm{CO}$ model grid along with the line ratios measured for the nucleus of M51 in Fig. 9. We also show the cold component density range calculated from our two-component RADEX fit overlaid on the low $\mathrm{J}$ line ratios (Fig. 9, left-hand column) and the warm component density range overlaid on the high $\mathrm{J}$ line ratios (Fig. 9, right-hand column). 


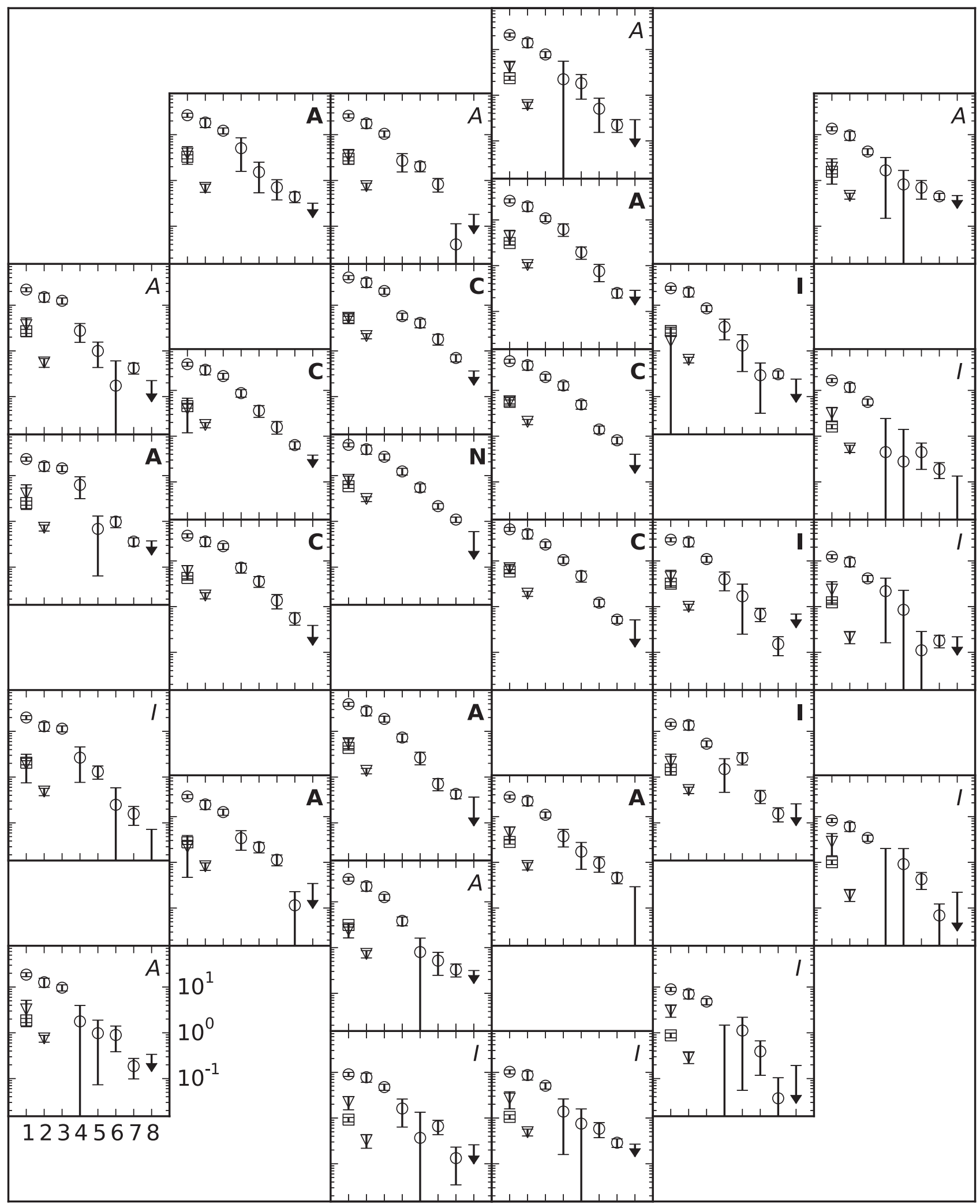

Figure 5. Measured $\mathrm{CO}$ (circles), [C I] (triangles) and ${ }^{13} \mathrm{CO}$ (squares) spectral line energy distribution in units of $\mathrm{K} \mathrm{km} \mathrm{s}^{-1}$ for each pixel in our FTS data cube. The $y$-axis corresponds to the integrated intensity in units of $\mathrm{K} \mathrm{km} \mathrm{s}^{-1}$ and the $x$-axis corresponds to $J_{\text {up }}$. The vertical and horizontal scales are the same for each box and are shown in the lower left-hand box. The CO J $=1-0$ to $\mathrm{J}=3-2$ and ${ }^{13} \mathrm{CO} \mathrm{J}=1-0$ transitions are from ground-based instruments (see Section 2.2.1). The error bars shown here do not include calibration uncertainties (see Table 1 for the calibration uncertainties). The letter in the upper right-hand corner indicates the pixel's region, where ' $\mathrm{N}$ ' corresponds to the nucleus, 'C' the centre, 'A' the arm and 'I' the interarm regions. Only the bolded letters are included in our region averages. 


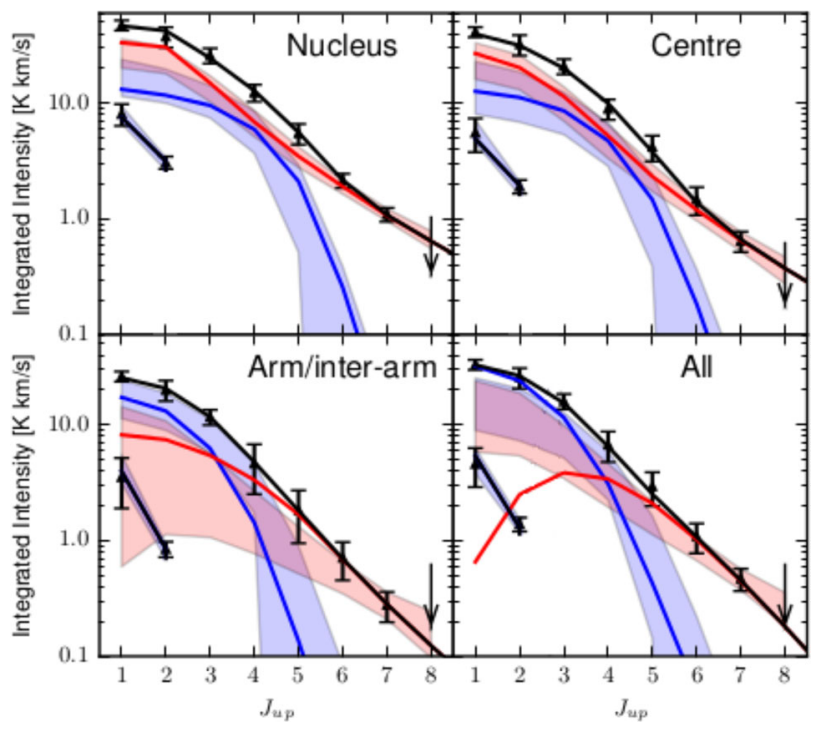

Figure 6. Measured and best-fitting SLEDs for the two-component fit for the nucleus (top-left), centre (top-right) and arm/interarm (bottom-left) regions, and for all the regions combined (bottom-right). The measured $\mathrm{CO}$ SLED (eight points) and [C I] SLED (two points) are shown by the black triangles with error bars. The cold component and warm component fits to the $\mathrm{CO}$ emission are shown by the blue and red lines, respectively, while the total calculated $\mathrm{CO}$ emission is shown by the solid black line. The cold component fit to the $\left[\mathrm{C}_{\mathrm{I}}\right]$ emission is indicated by a solid black line. For the cold and warm component fits, the coloured shaded regions indicate the $1 \sigma$ uncertainty region of the fits.

The $\mathrm{CO}$ ratios alone are unable to constrain both the density and FUV field strength (Fig. 9). The ratio of $L_{\mathrm{CO}} / L_{\mathrm{TIR}}$ provides an upper limit to the FUV field strength. We calculate a grid of the ratio of $L_{\mathrm{COJ}=3-2} / L_{\mathrm{TIR}}$ and $L_{\mathrm{COJ}=6-5} / L_{\mathrm{TIR}}$ by estimating $L_{\mathrm{TIR}}$ as twice the FUV field strength (Kaufman et al. 1999). We show the resulting grid in the bottom panel of Fig. 9, along with the measured ratio for the nucleus of M51. We correct the measured CO-to-TIR line ratios by multiplying the ratio by 2 to account for the fact that the measured CO emission from the PDR model is only from one side of the PDR, while the TIR emission as estimated is assumed to originate from both sides of the PDR. We show the measured $L_{\mathrm{COJ}=3-2} / L_{\mathrm{TIR}}$ ratios for the remaining regions in Fig. 10 , and the $L_{\mathrm{CO}(6-5)} / L_{\mathrm{TIR}}$ ratios in Fig. 11.

For the low-J CO line ratio PDR model, the CO line ratios coupled with the ratio of $L_{\mathrm{COJ}=3-2} / L_{\mathrm{TIR}}$ constrain the field strength to $G_{0}<10^{2}$ in all the modelled regions, while the PDR model density agrees with the low end of the range of density from our non-LTE analysis $\left(n\left(\mathrm{H}_{2}\right) \sim 10^{3}-10^{3.5} \mathrm{~cm}^{-3}\right.$, Fig. 10). For the high-J $\mathrm{CO}$ line ratio PDR model, the $\mathrm{CO}$ line ratios limit the density to
Table 3. Non-LTE model constraints.

\begin{tabular}{lcc}
\hline Parameter & Value & Units \\
\hline CO abundance $\left(x_{\mathrm{CO}}\right)$ & $3 \times 10^{-4}$ & $\ldots$ \\
Mean molecular weight $(\mu)$ & 1.5 & $\ldots$ \\
Angular size scale & 48.9 & $\mathrm{pc} \mathrm{arcsec}$ \\
Source size & 40 & $\operatorname{arcsec}$ \\
Length $(L)$ & $\leq 160$ & $\mathrm{pc}$ \\
\hline
\end{tabular}

$n\left(\mathrm{H}_{2}\right) \gtrsim 10^{4} \mathrm{~cm}^{-3}$, while the ratio of $L_{\mathrm{COJ}=6-5} / L_{\mathrm{TIR}}$ limits the field strength to $G_{0} \lesssim 10^{3}$ for all the modelled regions (Fig. 11). For the nucleus and centre regions, these densities are significantly higher than the densities calculated from our non-LTE analysis $\left(n\left(\mathrm{H}_{2}\right) \sim 10^{2.5}-10^{3.5} \mathrm{~cm}^{-3}\right)$ and so these PDR models do not provide a good fit to the data. For the arm/interarm region, both low $\left(n\left(\mathrm{H}_{2}\right) \sim 10^{2} \mathrm{~cm}^{-3}\right)$ and high $\left(n\left(\mathrm{H}_{2}\right) \sim 10^{4} \mathrm{~cm}^{-3}\right)$ density solutions exist; tighter constraints on the density are required to distinguish between the two solutions.

\section{DISCUSSION}

\subsection{Physical state of the molecular gas}

\subsubsection{Regional variations}

The physical state of both the cold and warm molecular gas can vary significantly from source to source, depending upon the environment in which the molecular gas resides. The results for the cold and warm components for the nucleus, centre and arm/interarm regions of M51 are shown in Table 4 and Fig. 8. For each of the components, no significant differences between regions are seen among any of the physical parameters. The density of the cold molecular gas in all three regions $\left(n\left(\mathrm{H}_{2}\right) \sim 10^{4} \mathrm{~cm}^{-3}\right)$ is typical for GMCs in the Milky Way (e.g. Tielens 2005), but is uncertain by three orders of magnitude (Table 4). The temperature of the cold component in all three regions is very similar to the typically quoted value of $\sim 10 \mathrm{~K}$ for Milky Way GMCs. Given the $1 \sigma$ ranges for the beam-averaged column densities in Table 4, any differences would need to be greater than a factor of $\sim 5$ ( $\sim 10$ for the arm/interarm region) to be seen in our results. Similarly for the warm component, we detect no differences between the temperature, density or beam-averaged column density across the three regions. The warm molecular gas has a temperature of $\sim 1000 \mathrm{~K}$. In the nucleus and centre, the density of the warm component is quite low $\left(n\left(\mathrm{H}_{2}\right) \sim 10^{1.6}-10^{2.5} \mathrm{~cm}^{-3}\right)$, while for the arm/interarm region the density is not very well constrained.

The similarities in the nucleus and centre of M51 are likely due to contamination of the beam at the nucleus position by emission from the centre region. Parkin et al. (2013) used data with a 12 arcsec beam and defined the nucleus to be a square $\sim 12$ arcsec across.

Table 2. RADEX grid parameters.

\begin{tabular}{lcc}
\hline Parameter & Range & Units \\
\hline Kinetic temperature, cold component $\left(T_{\text {kin }}\right.$, cold $)$ & $10^{0.5}-10^{1.5}$ & $\mathrm{~K}$ \\
Kinetic temperature, warm component $\left(T_{\text {kin }}\right.$, warm $)$ & $10^{0.7}-10^{3.8}$ & $\mathrm{~K}$ \\
$\mathrm{H}_{2}$ density $\left(n\left(\mathrm{H}_{2}\right)\right)$ & $10^{1.0}-10^{7.0}$ & $\mathrm{~cm}^{-3}$ \\
Area filling factor $\left(\Phi_{\mathrm{A}}\right)$ & $10^{-5.0}-1$ & $\ldots$ \\
$\mathrm{CO}$ column density per unit line width $\left(N_{\mathrm{CO}} / \Delta V\right)^{a}$ & $10^{12.0}-10^{18.0}$ & $\mathrm{~cm}^{-2}\left(\mathrm{~km} \mathrm{~s}^{-1}\right)^{-1}$ \\
{$\left[\mathrm{C}\right.$ i] $/ \mathrm{CO}$ column density ratio $\left(N_{[\mathrm{C}]} / N_{\mathrm{CO}}\right)$} & $10^{-2.0}-10^{2.0}$ & $\ldots$ \\
Line width $(\Delta V)$ & 1.0 & $\mathrm{~km} \mathrm{~s}^{-1}$ \\
\hline
\end{tabular}

Note. ${ }^{a}$ Column density is calculated per unit line width, while the line width is held fixed at $1 \mathrm{~km} \mathrm{~s}^{-1}$ in the calculations (see text). 
Table 4. Results from two-component non-LTE excitation analysis.

\begin{tabular}{lllll}
\hline & & \multicolumn{2}{c}{ Median $(-1 \sigma \rightarrow+1 \sigma ;$ best fit $)$} \\
Parameter ${ }^{a}$ & Nucleus & Centre & All & \\
\hline $\operatorname{Cold}$ component & & & & \\
$\log \left(T_{\text {kin }}\right)$ & $1.1(1.0 \rightarrow 1.3 ; 1.3)$ & $1.1(1.0 \rightarrow 1.3 ; 1.4)$ & $1.0(0.9 \rightarrow 1.4 ; 1.3)$ & $1.1(0.9 \rightarrow 1.4 ; 1.5)$ \\
$\log \left(n\left(\mathrm{H}_{2}\right)\right)$ & $4.0(3.0 \rightarrow 5.7 ; 3.6)$ & $4.3(3.0 \rightarrow 6.1 ; 3.4)$ & $4.3(2.9 \rightarrow 6.1 ; 3.0)$ & $4.1(2.9 \rightarrow 6.0 ; 2.4)$ \\
$\log \left(N_{\mathrm{CO}}\right)$ & $19.1(18.6 \rightarrow 19.5 ; 19.1)$ & $19.0(18.6 \rightarrow 19.4 ; 19.0)$ & $18.8(18.3 \rightarrow 19.2 ; 18.4)$ & $18.9(18.4 \rightarrow 19.3 ; 18.8)$ \\
$\log \left(\Phi_{\mathrm{A}}\right)$ & $-1.8(-2.1 \rightarrow-1.6 ;-2.1)$ & $-1.8(-2.1 \rightarrow-1.5 ;-2.2)$ & $-1.5(-1.8 \rightarrow-1.1 ;-1.7)$ & $-1.6(-2.0 \rightarrow-1.4 ;-1.5)$ \\
$\log \left(\left\langle N_{\mathrm{CO}}\right\rangle\right)$ & $17.2(16.8 \rightarrow 17.6 ; 17.0)$ & $17.2(16.8 \rightarrow 17.6 ; 16.8)$ & $17.3(16.8 \rightarrow 17.8 ; 16.7)$ & $17.2(16.8 \rightarrow 17.6 ; 17.3)$ \\
$\log (P)$ & $5.2(4.2 \rightarrow 6.7 ; 4.9)$ & $5.3(4.3 \rightarrow 7.1 ; 4.7)$ & $5.3(4.2 \rightarrow 7.0 ; 4.3)$ & $5.1(4.2 \rightarrow 6.9 ; 3.9)$ \\
$\log \left(N_{\mathrm{CC}]} / N_{\mathrm{CO}}\right)$ & $1.2(0.4 \rightarrow 1.7 ; 0.4)$ & $1.0(0.1 \rightarrow 1.6 ; 0.2)$ & $1.0(-0.1 \rightarrow 1.6 ; 0.2)$ & $0.9(0.0 \rightarrow 1.6 ;-0.2)$ \\
$\operatorname{Warm} \operatorname{component}$ & & & & \\
$\log \left(T_{\text {kin }}\right)$ & $3.2(2.8 \rightarrow 3.5 ; 3.0)$ & $2.9(2.5 \rightarrow 3.3 ; 2.9)$ & $2.8(2.2 \rightarrow 3.4 ; 2.4)$ & $2.8(2.4 \rightarrow 3.4 ; 2.1)$ \\
$\log \left(n\left(\mathrm{H}_{2}\right)\right)$ & $2.1(1.7 \rightarrow 2.4 ; 2.4)$ & $2.1(1.6 \rightarrow 2.5 ; 1.9)$ & $2.5(1.7 \rightarrow 4.5 ; 2.4)$ & $2.3(1.7 \rightarrow 3.3 ; 4.1)$ \\
$\log \left(N_{\mathrm{CO}}\right)$ & $17.9(16.9 \rightarrow 18.7 ; 16.5)$ & $17.8(16.9 \rightarrow 18.6 ; 18.4)$ & $17.6(16.3 \rightarrow 18.6 ; 18.8)$ & $17.7(16.6 \rightarrow 18.6 ; 16.6)$ \\
$\log \left(\Phi_{\mathrm{A}}\right)$ & $-1.4(-1.8 \rightarrow-0.5 ;-0.1)$ & $-1.3(-1.8 \rightarrow-0.6 ;-1.6)$ & $-1.7(-3.0 \rightarrow-0.7 ;-2.4)$ & $-1.4(-2.2 \rightarrow-0.6 ;-1.4)$ \\
$\log \left(\left\langle N_{\mathrm{CO}}\right\rangle\right)$ & $16.5(16.3 \rightarrow 16.9 ; 16.4)$ & $16.5(16.3 \rightarrow 16.9 ; 16.8)$ & $15.9(14.6 \rightarrow 16.4 ; 16.4)$ & $16.3(15.5 \rightarrow 16.7 ; 15.2)$ \\
$\log (P)$ & $5.3(4.9 \rightarrow 5.6 ; 5.4)$ & $5.1(4.6 \rightarrow 5.3 ; 4.7)$ & $5.3(4.8 \rightarrow 6.9 ; 4.8)$ & $5.1(4.7 \rightarrow 6.0 ; 6.2)$ \\
\hline
\end{tabular}

Note. ${ }^{a}$ Units for the parameters are as follows: $\left(T_{\mathrm{kin}}\right): \mathrm{K} ;\left(n\left(\mathrm{H}_{2}\right)\right): \mathrm{cm}^{-3} ;\left(N_{\mathrm{CO}}\right),\left(\left\langle N_{\mathrm{CO}}\right\rangle\right): \mathrm{cm}^{-2} ;(P): \mathrm{K} \mathrm{cm}^{-3} ;\left(\Phi_{\mathrm{A}}\right),\left(N_{[\mathrm{CI}]} / N_{\mathrm{CO}}\right):$ dimensionless.

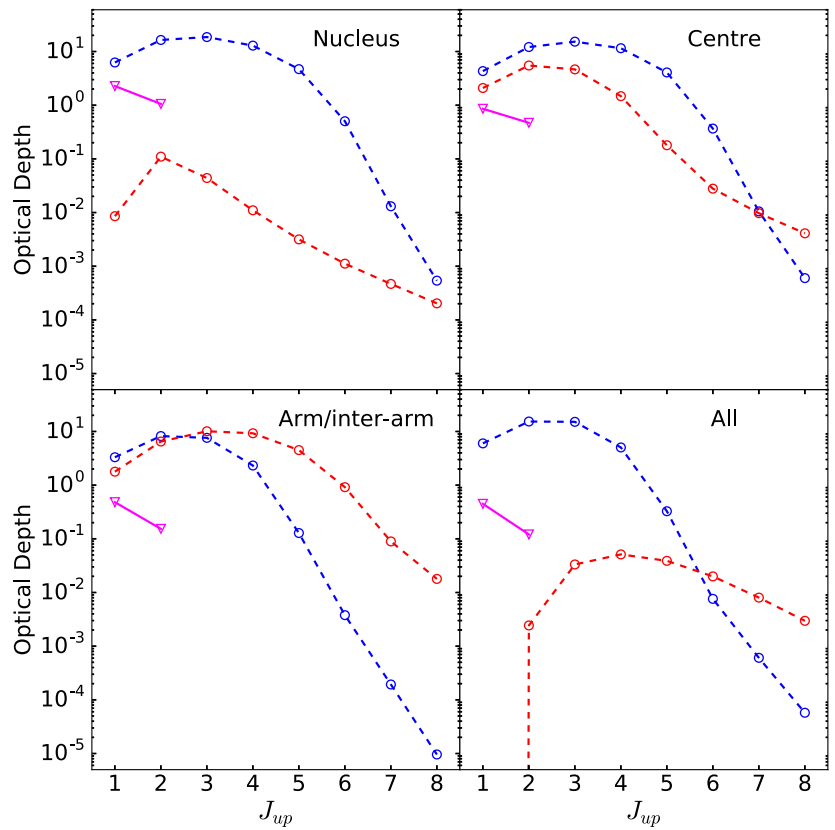

Figure 7. Best-fitting line optical depths for the two-component fit for the nucleus (top-left), centre (top-right) and arm/interarm (bottom-left) regions, and for all the regions combined (bottom-right). The cold component and warm component $\mathrm{CO}$ optical depths are shown by the blue and red dashed lines and circles, respectively. The [C I] optical depths are indicated by the solid magenta line and triangles.

Given the 40 arcsec beam of our observations, our nucleus region will be somewhat contaminated by emission from the centre region, and vice versa.

\subsubsection{Comparison to previous studies}

Both Israel et al. (2006) and Schinnerer et al. (2010) modelled various ratios of $\mathrm{CO}$ and ${ }^{13} \mathrm{CO}$ in M51. Schinnerer et al. (2010) used a non-LTE analysis to model ratios of ${ }^{12} \mathrm{CO} \mathrm{J}=1-0$ and $\mathrm{J}=2-1$, and ${ }^{13} \mathrm{CO} \mathrm{J}=1-0$ at multiple positions of the western arm and southern regions of M51 at resolutions of 2.9 and 4.5 arcsec; however, these regions lie beyond our FTS maps. They recovered cold $(14-20 \mathrm{~K})$, moderately dense $\left(n\left(\mathrm{H}_{2}\right) \sim 10^{2}-10^{2.4} \mathrm{~cm}^{-3}\right)$ gas.

Israel et al. (2006) modelled ${ }^{12} \mathrm{CO} \mathrm{J}=1-0$ to $\mathrm{J}=4-3$ and ${ }^{13} \mathrm{CO} \mathrm{J}=1-0$ to $\mathrm{J}=3-2$ line ratios at two locations in M51: the centre and in a giant molecular association (GMA) offset from the nucleus $\Delta \alpha=-10 \operatorname{arcsec}, \Delta \delta=+15$ arcsec. Given the small size of the offset and our large beam, both of these positions correspond to our nucleus. Using a Large Velocity Gradient (LVG) model, they fit two components to the $\mathrm{CO}$ line ratios, assuming that $\left[{ }^{12} \mathrm{CO}\right] /\left[{ }^{13} \mathrm{CO}\right]=40$. For the offset GMA, they find a warm $(\sim 100 \mathrm{~K})$, relatively diffuse $\left(\sim 10^{2.0} \mathrm{~cm}^{-3}\right)$ component, and a warmer $(\sim 150 \mathrm{~K})$, more dense $\left(\sim 10^{3.0} \mathrm{~cm}^{-3}\right)$ component. For the centre, they find a relatively warm $(\sim 100-1000 \mathrm{~K})$, lower density $\left(\sim 10^{2.0}-10^{3.0} \mathrm{~cm}^{-3}\right)$ component, and a cooler $(\sim 20-60 \mathrm{~K})$, higher density $\left(10^{3.0}-10^{3.5} \mathrm{~cm}^{-3}\right)$ component. The cold component from our two-component fit agrees with their centre results in both density and temperature, although our models do not constrain the density particularly well. Our warm component fit also agrees with their centre results in both density and temperature. Unlike Israel et al. (2006), we have observations of CO beyond the $\mathrm{J}=4-3$ transition and so are able to place significantly tighter constraints on the temperature of the warm component.

Brunner et al. (2008) probed the warm and hot molecular gas in M51 using the mid-infrared $\mathrm{H}_{2}$ rotational transitions $S(0)-S(5)$ in a strip. They found that the low-J $\mathrm{H}_{2}$ transitions $(S(0)-S(2))$ trace warm $(\sim 100-300 \mathrm{~K})$ molecular gas, while the high-J $\mathrm{H}_{2}$ transitions $(S(2)-S(5))$ trace hot molecular gas $(\sim 400-1000 \mathrm{~K})$. The temperature ranges for the warm and hot $\mathrm{H}_{2}$ gas agree quite well with the warm component from our fit to the $\mathrm{CO}$ data.

\subsubsection{Diffuse molecular gas}

As part of the PAWS collaboration, Pety et al. (2013) mapped the $\mathrm{CO} \mathrm{J}=1-0$ emission in M51 at arcsecond resolution. By combining the PdBI interferometric data with the IRAM 30-m data, they were able to correct for the 'missing flux' from the interferometric observations. They find that $\sim 50$ per cent of the $\mathrm{CO} J=1-0$ emission is from molecular gas located in a thick, extended disc with a scale height $\sim 200$ pc. Pety et al. (2013) argue that this emission 


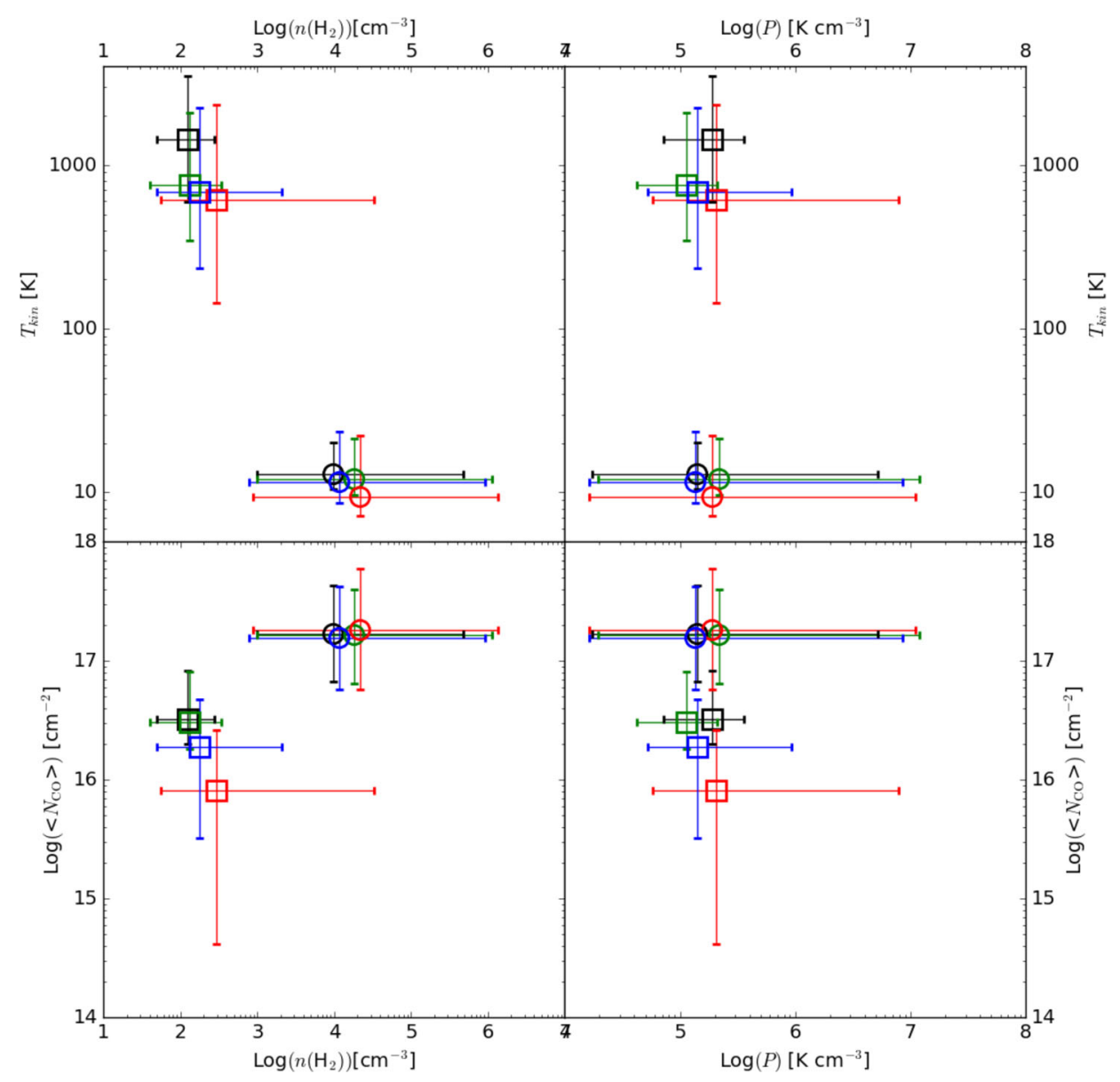

Figure 8. Derived physical parameters for the cold (circles) and warm (squares) components of the multicomponent RADEX model averaged over the nucleus (black), centre (green) and arm/interarm (red) regions of M51 (see Fig. 2 and Section 3 for more details), and for the average of all the regions combined (blue). The symbols are plotted at the location of the median solution for each parameter. $\mathrm{CO}$ is included in both the cold and warm components, while [C $\mathrm{I}$ ] is included only in the cold component. The error bars correspond to the $1 \sigma$ range of the combined likelihood distribution of each region for the kinetic temperature (top row), beam-averaged column density (bottom row), molecular gas density (left-hand column) and pressure (right-hand column).

originates from a warm $(\sim 50-100 \mathrm{~K})$, diffuse $\left(\sim 100-500 \mathrm{~cm}^{-3}\right)$ molecular component.

We investigate the possible effects of this extended $\mathrm{CO}$ emission on our two-component non-LTE model by fitting various combinations of the $\mathrm{CO}$ and $\left[\mathrm{C}_{\mathrm{I}}\right]$ transitions (Table 5). We begin by setting the $\mathrm{CO} \mathrm{J}=1-0$ flux to half of the measured flux and fitting it along with the remaining $\mathrm{CO}$ and $\left[\mathrm{C}_{\mathrm{I}}\right]$ transitions as before (half10). Additionally, to investigate any contributions from the warm, diffuse component to the $\mathrm{CO} \mathrm{J}=2-1$ transition, we set the $\mathrm{CO} \mathrm{J}=2-1$ transition as an upper limit while fitting half of the CO $\mathrm{J}=1-0$ flux (half10 2ul). We compare these solutions to our original twocomponent solutions (all lines) for the centre region in Table 5. For the cold component, the $1 \sigma$ ranges for the kinetic temperatures do not shift significantly among the models, while the $1 \sigma$ ranges for the molecular gas density narrow slightly for both the half10 and half10 $2 u l$ solutions compared to the all lines solution. However, the half10 and half10 $2 \mathrm{ul}$ warm component solutions have significantly higher densities, higher pressures and lower beam-averaged column densities compared to the all lines solution.

The changes in the warm component density for both the half10 and half10 $2 u l$ solutions suggest that the diffuse molecular gas component from Pety et al. (2013) contributes to the CO J $=1-0$ emis- sion, and possibly the $\mathrm{CO} \mathrm{J}=2-1$ emission. However, determining the physical characteristics of this component using a non-LTE model, such as the one presented in Section 3, would be difficult. The CO J $=1-0$ emission from the diffuse, extended component is subthermally excited and there would be even less contribution from the diffuse component to higher J CO transitions. While Pety et al. (2013) use the ratio of $\frac{{ }^{12} \mathrm{CO}}{{ }^{13} \mathrm{CO}}$ to argue for the existence of this diffuse component, including only a single ${ }^{13} \mathrm{CO}$ transition in a non-LTE analysis requires assuming a relative abundance ratio of ${ }^{12} \mathrm{CO}$ and ${ }^{13} \mathrm{CO}$ in the diffuse component.

The existence of an extended, diffuse component does not preclude the diffuse molecular gas, at least in part, being contained within GMCs. Diffuse GMCs exhibiting high ratios of $\frac{{ }^{12} \mathrm{CO}}{{ }^{13} \mathrm{CO}}$ have been observed at high latitudes within our own Galaxy (Blitz, Magnani \& Mundy 1984). In this scenario, the limited sensitivity of the interferometer along with the unresolved nature of the brightest clumps of the diffuse GMCs would lead to the interferometer filtering out these diffuse GMCs (Pety et al. 2013).

The critical densities of the $\left[\mathrm{C}_{\mathrm{I}}\right] \mathrm{J}=1-0$ and $\mathrm{J}=2-1$ transitions are $n_{10} \sim 500 \mathrm{~cm}^{-3}$ and $n_{21} \sim 10^{3} \mathrm{~cm}^{-3}$, respectively (Papadopoulos et al. 2004). These are comparable to the critical density of $\mathrm{CO} \mathrm{J}=1-0\left(n_{\mathrm{cr}} \sim 1.1 \times 10^{3} \mathrm{~cm}^{-3}\right)$, which indicates that $[\mathrm{C} \mathrm{I}]$ 


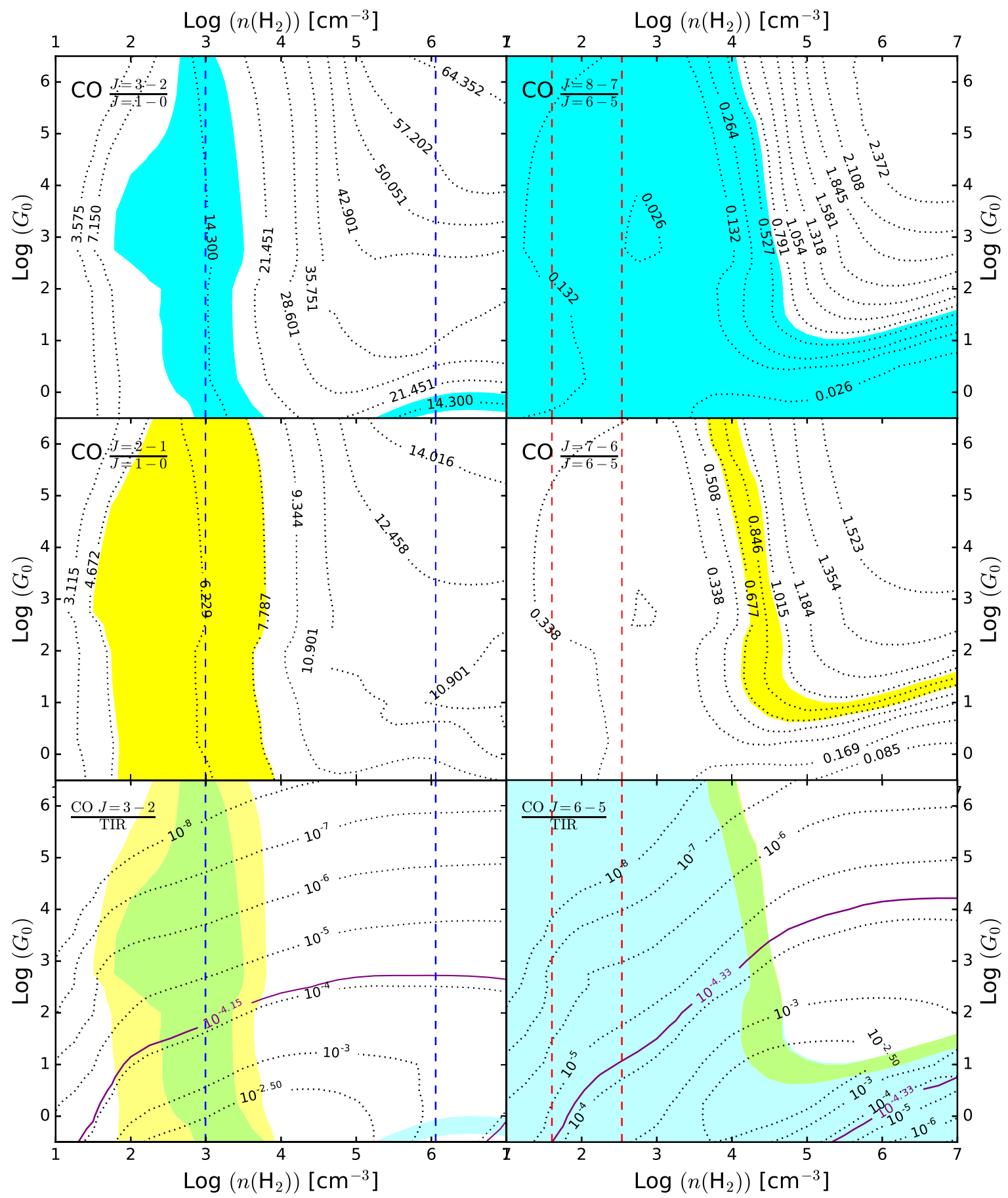

Figure 9. PDR model line ratios for $\mathrm{CO} \frac{\mathrm{J}=3-2}{\mathrm{~J}=1-0}$ (top-left), $\frac{\mathrm{J}=2-1}{\mathrm{~J}=1-0}$ (middle-left), $\frac{\mathrm{J}=8-7}{\mathrm{~J}=6-5}$ (top-right) and $\frac{\mathrm{J}=7-6}{\mathrm{~J}=6-5}$ (middle-right) for the nucleus of M51 in units of $\mathrm{W} \mathrm{m}^{-2}$. Note that the ratio of $\frac{\mathrm{J}=8-7}{\mathrm{~J}=6-5}$ is an upper limit. In the top two rows, the dotted contours correspond to constant CO line ratios. The blue (top-row) and yellow (middle-row) shaded regions correspond to the uncertainty in the measured line ratio for the nucleus. In the bottom row, the dotted contours correspond to constant value of $\frac{L_{\mathrm{COJ}=3-2}}{L_{\mathrm{TIR}}}$ (bottom-left) and $\frac{L_{\mathrm{CO} J=6-5}}{L_{\mathrm{TTR}}}$ (bottom-right), while the solid purple line is the measured ratio for each. The shaded regions in the bottom-row panels correspond to the same CO line ratios of the two panels directly above, while the green region indicates where the two line ratios overlap. The blue (left-hand column) and red (right-hand column) dashed vertical lines correspond to the cold and warm component $1 \sigma$ ranges for the densities from the two-component RADEX solutions.

could also, at least in part, be tracing a diffuse molecular component. High-resolution observations of [C $\mathrm{I}]$ in galaxies like M51 coupled with single-dish observations, as for CO in Pety et al. (2013), may be useful in constraining the physical state of this diffuse gas. Combining such observations with interferometric observations of dense gas tracers, such as $\mathrm{HCN}$ and $\mathrm{HCO}+$, would allow us to discriminate between dense and diffuse GMCs. Finally, high-sensitivity, flux-recovered observations of a combination of 
these molecular gas-tracing species using the Atacama Large Millimeter Array (ALMA) would help differentiate between a truly extended component or a collection of diffuse GMCs. Unfortunately, M51 itself is not a viable target for ALMA due to its high declination.

\subsection{PDR modelling}

Our comparison of the PDR and non-LTE models suggests that the warm component of the molecular gas in the nucleus and centre of M51 is unlikely to reside primarily in simple PDRs. The LINER nucleus (Satyapal et al. 2004) may produce a small X-ray-dominated region that could affect the excitation in the vicinity of the nucleus. On larger scales, Kazandjian et al. (2015) have shown that a relatively small amount of mechanical heating from supernovae and stellar winds can have significant effects on the temperature of the molecular gas. The presence of active star formation in M51 certainly suggests that supernovae will be present in the disc. However, we cannot easily estimate the mechanical heating due to supernovae and stellar winds for M51 as the necessary supernova rate data do not exist. The strong spiral density wave in M51 may also create shocks that are themselves a potential source of mechanical heating. We also note that our efforts to estimate the effect of the diffuse molecular component proposed by Pety et al. (2013) result in models with higher warm component densities that would be consistent with PDR models. We therefore focus our discussion in this section on the cold component of the molecular gas, for which the PDR models are able to reproduce the $\mathrm{CO}$ data.

Both Kramer et al. (2005) and Parkin et al. (2013) previously modelled PDRs in M51. Kramer et al. (2005) modelled various ratios of $\left[\mathrm{C}_{\mathrm{II}}\right](158 \mu \mathrm{m}),\left[\mathrm{O}_{\mathrm{I}}\right](63 \mu \mathrm{m}),\left[\mathrm{C}_{\mathrm{I}}\right] \mathrm{J}=1-0$, $\mathrm{CO} \mathrm{J}=1-0$ and $\mathrm{CO} \mathrm{J}=3-2$ using the PDR models from Kaufman et al. (1999). They found that the best-fitting solution to their line ratios at all three pointings was consistent with density of $n\left(\mathrm{H}_{2}\right) \sim 4 \times 10^{4} \mathrm{~cm}^{-3}$ and a field strength of $18<G_{0}<32$ in an $80 \mathrm{arcsec}$ beam. While their value of $G_{0}$ is comparable to the results for our cold component, their value for the density is an order of magnitude larger than our result.

Parkin et al. (2013) used the $\left[\mathrm{C}_{\mathrm{II}}\right] /\left[\mathrm{O}_{\mathrm{I}}\right] 63 \mu \mathrm{m}$ and $\left(\left[\mathrm{C}_{\mathrm{II}}\right]+\right.$ [OI] $63 \mu \mathrm{m}$ )/TIR ratios along with the PDR models from Kaufman et al. (1999) and Kaufman, Wolfire \& Hollenbach (2006) to constrain the density and field strength in the nucleus, centre, arm and interarm regions of M51 (see Fig. 2). They corrected the [C II] emission for the ionized gas fraction and [O I] $(63 \mu \mathrm{m})$ for orientation effects due to the plane-parallel slab-nature of the models (see section 4.1 of Parkin et al. 2013 for details). Their results for the nucleus $\left(n\left(\mathrm{H}_{2}\right)=10^{3.75}-10^{4.0} \mathrm{~cm}^{-3}, G_{0}=10^{3.25}-10^{3.75}\right)$ do not agree with our 'cold' PDR solution, falling above the range allowed by our CO line ratios and our CO/TIR ratios. Their density for the centre $\left(n\left(\mathrm{H}_{2}\right)=10^{3.0}-10^{3.25} \mathrm{~cm}^{-3}\right)$ agrees with our 'cold' PDR solution density, while their field strength $\left(G_{0}=10^{2.75}-10^{3.0}\right)$ exceeds what is allowed by our ratio of $\mathrm{CO} / \mathrm{TIR}$ combined with various $\mathrm{CO}$ line ratios (Fig. 10). Their results for both the arm and interarm regions are similar: the ranges reported for the density $\left(n\left(\mathrm{H}_{2}\right)=10^{2.75}\right.$ $10^{3.0} \mathrm{~cm}^{-3}$ ) agree with our results, while the FUV field strength $\left(G_{0}=10^{2.25}-10^{2.5}\right)$ is larger than our allowed solutions.

Our 'cold' PDR results suggest a density of $n\left(\mathrm{H}_{2}\right) \sim 10^{3} \mathrm{~cm}^{-3}$ and FUV field strength of $G_{0}<10^{2}$. In the Kaufman et al. (1999, 2006) PDR models, this would correspond to $\frac{[\mathrm{CII}]}{[\mathrm{OI}](63 \mu \mathrm{m})} \gtrsim 1.7$ and $\frac{[\mathrm{CII}]+[\mathrm{OI}](63 \mu \mathrm{m})}{\mathrm{TIR}} \gtrsim 1.3 \times 10^{-2}$. The average values of these ratios in each of the nucleus, centre, arm and interarm regions

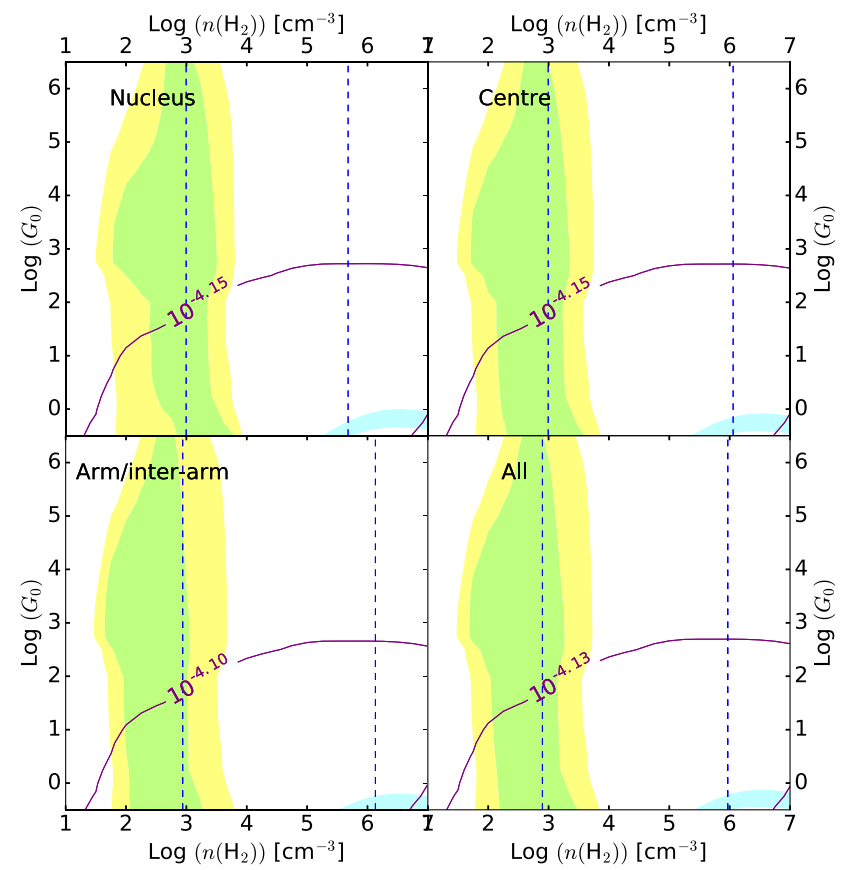

Figure 10. Same as the bottom left-hand panel of Fig. 9 except for the cold component PDR solutions for the nucleus (top-left), centre (top-right) and arm/interarm (bottom-left) regions of M51, and for all four regions combined (bottom-right). Note that the line ratios are calculated in units of $\mathrm{W} \mathrm{m}{ }^{-2}$.

from Parkin et al. (2013) vary between $\frac{[\mathrm{C} \mathrm{II}]}{[\mathrm{OI}(63 \mu \mathrm{m})} \sim 0.2-1.2$ and $\frac{[\mathrm{CII}]+[\mathrm{OI}](63 \mu \mathrm{m})}{\mathrm{TIR}} \sim(5.0-8.1) \times 10^{-3}$. For both ratios, the values for our 'cold' PDR solution are a factor of $\sim 1.5$ greater than the values measured in Parkin et al. (2013). Smoothing the Parkin et al. (2013) data to our 40 arcsec beam and remeasuring these ratios does not change the average values significantly. However, including the $\sim 30$ per cent calibration uncertainty of the PACS spectrometer ${ }^{5}$ leads to a range of $\frac{[\mathrm{CII}]+[\mathrm{OI}](63 \mu \mathrm{m})}{\mathrm{TIR}} \sim\left(3.5-10.5 \times 10^{-3}\right)$, which agrees within 50 per cent with the range necessary to reproduce our results using the Kaufman et al. (2006) PDR models.

Accounting for the calibration uncertainty leads to a range of $\sim 0.4-1.8$ for the ratio of $\frac{[\mathrm{CII}]}{[\mathrm{OI}](63 \mu \mathrm{m})}$, which agrees within uncertainties with the model value of $>1.7$.

We attribute the remaining disagreement to the simplified geometries assumed by the PDR models, which can lead to differences in the model results (Röllig et al. 2007). Both the model used in Parkin et al. (2013) and the model used here assume a semi-infinite slab of gas illuminated by an FUV field. In reality, PDRs are clumpy media that are affected by other physical processes concurrently. Mechanical heating, for example, can have a significant effect on the atomic and ion line ratios, as well as on the overall shape of the CO SLED (Kazandjian et al. 2015). Combining the measurements from Parkin et al. (2013) and our measured CO SLED, along with observations of other molecular gas tracers such as $\mathrm{HCN}$ and $\mathrm{HCO}+$, would be necessary to quantify the contributions of mechanical heating to the PDRs in M51.

${ }^{5}$ PACS Observers Manual is available for download from the ESA Herschel Science Centre. 


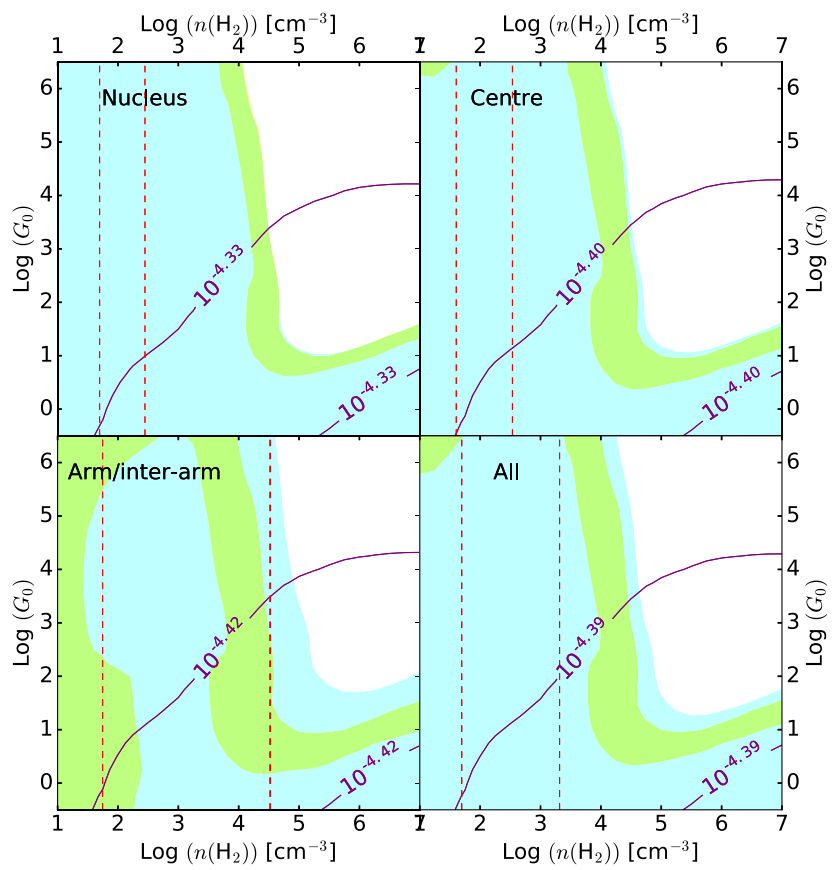

Figure 11. Same as the bottom right-hand panel of Fig. 9 except for the warm component PDR solutions for the nucleus (top-left), centre (top-right) and arm/interarm (bottom-left) regions of M51, and for all four regions combined (bottom-right). Note that the line ratios are calculated in units of $\mathrm{W} \mathrm{m}{ }^{-2}$.

\subsection{Comparison to other VNGS galaxies}

M51 is the sixth galaxy in the VNGS sample for which the analysis of the SPIRE-FTS observations of $\mathrm{CO}$ has been published, and is the first normal, quiescent spiral galaxy from the sample. Only a single component was fit to the CO SLED for M83 (Wu et al. 2015), while for NGC 1068 (Spinoglio et al. 2012), the extended (star-forming ring) and compact (circumnuclear disc) $\mathrm{CO}$ emission were fit separately, taking advantage of the varying beam size of the SLW and SSW. In the case of Arp 220 (Rangwala et al. 2011), M82 (Panuzzo et al. 2010; Kamenetzky et al. 2012) and NGC 4038/39 (Schirm et al. 2014), a two-component fit was performed for the CO, ${ }^{13} \mathrm{CO}(\mathrm{M} 82)$ and [C I](NGC 4038/39) emission. Arp 220, M82 and NGC 4038/39 are all examples of either an interaction or an ongoing merger. Arp 220 ( $D=77 \mathrm{Mpc}$; Scoville, Yun \& Bryant 1997) is an ultraluminous infrared galaxy (ULIRG) and is an advanced merger between two galaxies. M82 ( $D=3.4 \mathrm{Mpc}$; Dalcanton et al. 2009) is a starburst galaxy (Yun et al. 1993) whose increased star formation rate is due to a recent interaction with the nearby M81. Finally, NGC 4038/39 ( $D=22 \mathrm{Mpc}$; Schweizer et al. 2008) is an ongoing merger between two gas-rich spiral galaxies.

We compare our two-component fit for the centre region of M51 to those of Arp 220, M82 and NGC 4038/39 (Table 6 and Fig. 12). We also compare our results to the mean values for the two-component fits of Kamenetzky et al. (2014) for 17 galaxies, including the five galaxies from the VNGS. For NGC 4038/39, we distinguish between the region where the two merging gas discs overlap (the 'overlap region') and the nucleus of NGC 4038 (hereafter NGC 4038). For NGC 4038, the physical size corresponding to the beam of the observations ( $\sim 43 \mathrm{arcsec})$ is $\sim 4.6 \mathrm{kpc}$. In comparison, our analysis of M51 covers the central $\sim 5 \mathrm{kpc}(\sim 100 \mathrm{arcsec})$. Note that for Arp 220, the CO emission is point like within the FTS beam (Rangwala et al. 2011) and the actual column density is reported in Table 6. [Rangwala et al. (2011) only report bestfitting values for the parameters for Arp 220 (rather than medians); however, the probability distributions for the Arp 220 fits are quite narrow and so the difference between median and best fit is likely small for this galaxy.]

The characteristics of the cold component for the centre region of M51 are very similar to the two regions in NGC 4038/39. Aside from the large temperature range for $\mathrm{M} 82\left(T_{\mathrm{kin}}=12-472 \mathrm{~K}\right)$, the cold molecular gas in M51 is also similar to that of M82. The temperature of the cold molecular gas in $\operatorname{Arp} 220\left(T_{\text {kin }}=34-67 \mathrm{~K}\right)$ is above the temperature range we adopted in fitting the cold component in M51. The cold component fits to the 17 galaxies from Kamenetzky et al. (2014) cover a similar range in temperature $\left(T_{\text {kin }}=12-250 \mathrm{~K}\right)$.

The warm component of M51 shows more differences than the cold component when compared to the other galaxies, with a lower density $\left(n\left(\mathrm{H}_{2}\right)=10^{1.6}-10^{2.5} \mathrm{~cm}^{-3}\right)$ than any of the other three systems $\left(10^{3}<n\left(\mathrm{H}_{2}\right)<10^{6.6} \mathrm{~cm}^{-3}\right)$. The warm component fits from Kamenetzky et al. (2014) have a similar temperature range to M51, but densities $\left(10^{2.3}<n\left(\mathrm{H}_{2}\right)<10^{4.9} \mathrm{~cm}^{-3}\right)$ that only just overlap the M51 solution.

The molecular gas mass is proportional to the beam size multiplied by the beam-averaged column density. Since the beam size in any one system will be the same for the cold and warm components, we can calculate the warm gas mass as a fraction of the cold gas mass using the beam-averaged column densities. In M51, the $1 \sigma$ range for the warm gas mass is 7-65 per cent (mean 20 per cent) of the cold gas mass for the nucleus and centre regions, and $0.2-$ 30 per cent (mean 3 per cent) for the arm/interarm region. Interestingly, these fractions are comparable to the warm gas mass fractions of $\sim 10$ per cent in Arp 220 (Rangwala et al. 2011) and $\sim 3$ per cent in M82 (Kamenetzky et al. 2012) but are significantly larger than

Table 5. Including diffuse gas in radiative transfer solutions for the centre region.

\begin{tabular}{|c|c|c|c|c|c|}
\hline \multirow[b]{2}{*}{ Component } & \multirow[b]{2}{*}{ Solution } & \multicolumn{4}{|c|}{$\begin{array}{c}\text { Parameter } \\
\text { Median ( } 1 \sigma \text { range })\end{array}$} \\
\hline & & $\begin{array}{l}\log \left(n\left(\mathrm{H}_{2}\right)\right) \\
{\left[\log \left(\mathrm{cm}^{-3}\right)\right]}\end{array}$ & $\begin{array}{l}\log \left(T_{\text {kin }}\right) \\
{[\log (\mathrm{K})]}\end{array}$ & $\begin{array}{l}\log \left(\left\langle N_{\mathrm{CO}}\right\rangle\right) \\
{\left[\log \left(\mathrm{cm}^{-2}\right)\right]}\end{array}$ & $\begin{array}{l}\log (P) \\
{\left[\log \left(\mathrm{K} \mathrm{cm}^{-2}\right)\right]}\end{array}$ \\
\hline \multirow[t]{3}{*}{ Cold } & all lines & $4.3(3.0-6.1)$ & $1.1(1.0-1.3)$ & $17.2(16.8-17.6)$ & $5.3(4.3-7.1)$ \\
\hline & half10 ${ }^{a}$ & $3.9(3.1-5.4)$ & $1.2(1.0-1.4)$ & $16.6(16.3-17.3)$ & $5.2(4.3-6.5)$ \\
\hline & half10 $2 u l^{b}$ & $4.0(3.1-5.7)$ & $1.2(1.0-1.4)$ & $17.0(16.5-17.6)$ & $5.2(4.4-6.8)$ \\
\hline Warm & half10 $2 u l^{b}$ & $3.2(2.7-4.4)$ & $2.5(2.0-3.1)$ & $15.7(15.0-16.1)$ & $5.8(5.5-6.8)$ \\
\hline
\end{tabular}

Notes. ${ }^{a} \mathrm{CO} \mathrm{J}=1-0$ set to half its observed value; see text.

${ }^{b} \mathrm{CO} \mathrm{J}=1-0$ set to half its observed value and $\mathrm{CO} \mathrm{J}=2-1$ treated as an upper limit; see text. 
Table 6. Comparing radiative transfer solutions.

\begin{tabular}{|c|c|c|c|c|c|c|}
\hline Comp. & Source & \multicolumn{4}{|c|}{$\begin{array}{c}\text { Parameter } \\
\text { Median ( } 1 \sigma \text { range })\end{array}$} & Reference \\
\hline \multirow{3}{*}{ Cold } & $\operatorname{Arp} 220^{a}$ & $2.8(2.6-3.2)$ & $50(34-67)$ & $20.3(19.9-20.3)$ & $4.5(4.5-4.8)$ & Rangwala et al. (2011) \\
\hline & M82 & $3.2(2.4-4.8)$ & $40(12-472)$ & $18.2(17.6-18.8)$ & $5.1(4.6-5.8)$ & Kamenetzky et al. (2012) \\
\hline & NGC 4038 & $3.7(3.1-4.3)$ & $24(18-33)$ & $17.1(16.6-17.6)$ & $5.0(4.5-5.6)$ & Schirm et al. (2014) \\
\hline \multirow[t]{5}{*}{ Warm } & Centre of M51 & $2.1(1.6-2.5)$ & $755(347-2089)$ & $16.5(16.3-16.9)$ & $5.1(4.6-5.3)$ & This work \\
\hline & $\operatorname{Arp} 220^{a}$ & $3.2(3.0-3.2)$ & $1343(1247-1624)$ & $19.4(19.4-19.5)$ & $6.3(6.2-6.4)$ & Rangwala et al. (2011) \\
\hline & M82 & $4.0(3.5-4.2)$ & $414(335-518)$ & $16.7(16.4-17.2)$ & $6.6(6.2-6.8)$ & Kamenetzky et al. (2012) \\
\hline & NGC 4038 & $5.8(4.8-6.6)$ & $1065(347-3397)$ & $14.4(14.2-14.8)$ & $9.3(8.2-10.2)$ & Schirm et al. (2014) \\
\hline & Overlap region & $4.2(4.0-4.7)$ & $1425(430-3811)$ & $14.6(14.4-14.7)$ & $7.4(7.3-7.7)$ & Schirm et al. (2014) \\
\hline
\end{tabular}

Note. ${ }^{a}$ 'Best-fitting' values and actual $N_{\mathrm{CO}}$ reported for Arp 220; see text.

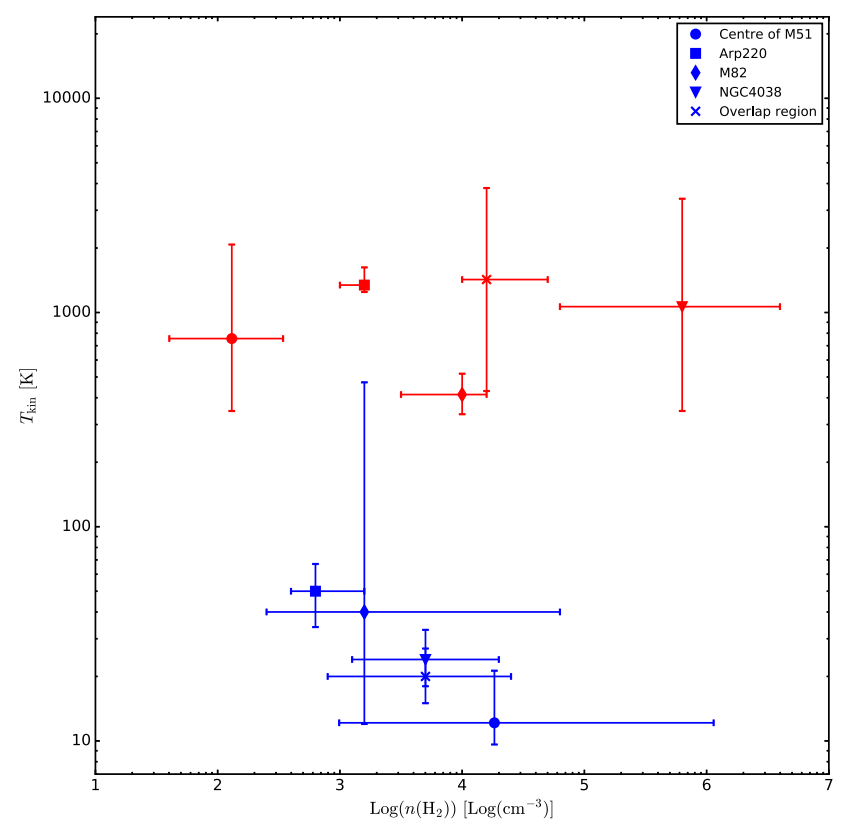

Figure 12. Comparison of results from the VNGS. The error bars represent the $1 \sigma$ ranges reported in Table 6 and the symbols are placed at the median of the distribution. The values for the temperature (y-axis) and molecular gas density ( $x$-axis) are the non-LTE excitation analysis results from this work (centre of M51), Rangwala et al. (2011) (Arp 220), Kamenetzky et al. (2012) (M82) and Schirm et al. (2014) (NGC 4038 and the overlap region). The cold and warm components are represented by the blue and red data points, respectively.

the warm gas mass fraction of $\sim 0.1$ per cent in NGC 4038 and the overlap region (Schirm et al. 2014). The average ratio of warm to cold gas mass in the Kamenetzky et al. (2014) sample is 12 per cent, with a range from 0.4 (for NGC 4038) to 40 per cent.

The global star formation rates in Arp $220\left(\sim 200 \mathrm{M}_{\odot} \mathrm{yr}^{-1}\right.$; Barcos-Muñoz et al. 2015) and M82 $\left(\sim 10 \mathrm{M}_{\odot} \mathrm{yr}^{-1}\right.$; Yun et al. 1993) are larger than the global star formation rates in M51 ( 2.6 $\mathrm{M}_{\odot} \mathrm{yr}^{-1}$; Schuster et al. 2007) and NGC 4038/39 ( $25 \mathrm{M}_{\odot} \mathrm{yr}^{-1}$; Stanford et al. 1990) by factors of 2-80. In addition, the gas and star formation rate surface densities are significantly higher in Arp 220 (Rangwala et al. 2011) and M82 (Kamenetzky et al. 2012), where the active regions are roughly $1 \mathrm{kpc}$ in size, than in M51 and the Antennae, where the activity is spread over the entire disc. The primary heating source for the warm molecular gas in
Arp 220 (Rangwala et al. 2011) and M82 (Kamenetzky et al. 2012) was found to be mechanical heating due primarily to supernova and stellar winds (Maloney 1999). The higher warm gas mass fraction in Arp 220 and M82 compared to NGC 4038/39 was attributed to an increase in the efficiency by which energy from supernova and stellar winds is injected as thermal energy into the molecular gas (Schirm et al. 2014). It is unclear whether stellar feedback can also explain the high warm gas mass fraction in M51. Perhaps shocks produced by the strong spiral density wave in M51 can provide an efficient source of turbulent energy.

The overlap region of NGC 4038/39 provides us with a comparison of the effects of an early stage major merger to the less pronounced interaction seen in M51. The most striking differences are in the density and pressure of the warm molecular gas, which are both roughly two orders of magnitude smaller in M51 compared to the overlap region of NGC 4038/9. In NGC 4038/39, supernova and stellar winds were found to be sufficient to heat the warm molecular gas (Schirm et al. 2014). For the densest gas, there is also evidence that PDRs with at least 5 per cent mechanical heating contribute to the overall heating budget (Schirm et al. 2016). In M51, there is strong evidence to suggest that PDRs are fundamental to the molecular gas heating (e.g. see Roussel et al. 2007; Parkin et al. 2013), although PDR models may not be sufficient to explain the heating of the warm component (Section 5.2). Both PDRs and mechanical heating, however, are tied to the star formation rate as both require the formation of $\mathrm{O}$ and $\mathrm{B}$ stars that are relatively short lived. A relatively small amount of mechanical heating compared to heating due to PDRs ( $\sim 1$ per cent) is able to produce a significant effect on the temperature of the molecular gas (e.g. Kazandjian et al. 2012). In NGC 4038/39, the turbulent motion due to both the ongoing merger and stellar feedback should exceed this minimum threshold (Schirm et al. 2014).

It is possible that similar effects are also at work in M51, which is itself an interacting system. Using more sophisticated PDR models that include contributions from mechanical heating, along with dense gas tracers such as $\mathrm{HCN}$ and $\mathrm{HCO}+$, may allow us to calculate the contributions to the total gas heating from PDRs and mechanical heating in M51, NGC 4038/39 and other systems.

\section{SUMMARY AND CONCLUSIONS}

In this paper, we have presented intermediate-sampled SPIRE-FTS observations of $\mathrm{CO}$ from $\mathrm{J}=4-3$ to $\mathrm{J}=8-7$ and both $\left[\mathrm{C}_{\mathrm{I}}\right]$ transitions of the central region of M51. We supplemented these observations 
with ground-based observations of $\mathrm{CO} \mathrm{J}=1-0$ to $\mathrm{J}=3-2$. We separate M51 into three regions, the nucleus, centre and arm/interarm regions, by performing an unweighted average of the emission for each pixel contained with a region. We also combine all the pixels within the three into a single 'All' region.

(i) Using the non-LTE excitation code RADEX along with a Bayesian likelihood code, we perform a two-component fit to the $\mathrm{CO}$ and $\left[\mathrm{C}_{\mathrm{I}}\right]$ emission in the nucleus, centre and arm/interarm regions of M51, along with all three regions combined. We find that the results do not vary beyond $1 \sigma$ for all three regions. The results for the nucleus and centre regions of M51 consist of a cold component $\left(T_{\text {kin }} \sim 10-20 \mathrm{~K}\right)$ with a moderate but poorly constrained density $\left(n\left(\mathrm{H}_{2}\right)=10^{3}-10^{6}\right)$ and a warm, somewhat lower density component $\left(T_{\text {kin }} \sim 300-3000 \mathrm{~K}, n\left(\mathrm{H}_{2}\right)=10^{1.6}-10^{2.5}\right)$. The results for the arm/interarm region, and for all regions combined are not as well constrained. The warm gas mass fraction for the centre of M51 is 20 per cent.

(ii) Pety et al. (2013) argue that $\sim 50$ per cent of the CO J $=1-$ 0 emission in M51 arises from warm diffuse gas. We investigate the possible effect of this extended component by re-running our models with the $\mathrm{CO} J=1-0$ flux set to half of the measured flux in our non-LTE analysis. The density range of the cold molecular gas narrows somewhat in these models, while the warm component shifts to significantly higher density and pressure. This analysis is consistent with the remaining $\mathrm{CO} J=1-0$ emission originating from a more diffuse, possibly extended component of the molecular gas. We suggest that this diffuse molecular gas may still arise from GMCs. High-resolution, high-sensitivity, flux-recovered observations of multiple molecular gas-tracing species, such as [C $\mathrm{I}]$, $\mathrm{HCN}$ and $\mathrm{HCO}+$ along with $\mathrm{CO}$, would allow us to distinguish between dense and diffuse GMCs.

(iii) We compare line ratios of $\mathrm{CO}$ along with the $\frac{\mathrm{COJ}=3-2}{\mathrm{TIR}}$ and $\frac{\mathrm{COJ}=6-5}{\mathrm{TIR}}$ ratios to a PDR model. Using the densities calculated from our non-LTE excitation analysis, our PDR modelling suggests a density of $n\left(\mathrm{H}_{2}\right) \sim 10^{3} \mathrm{~cm}^{-3}$ and a field strength $G_{0}<10^{2}$ for the cold component. Although the warm component in the arm/interarm regions is consistent with PDR heating, additional heating sources beyond PDRs seem to be required for the nucleus and centre regions. We compare our results to previous results (Parkin et al. 2013), which used various atomic line ratios and the total infrared flux to model the FUV field strength and gas density, and find that their FUV field strength $\left(G_{0}>10^{2.25}\right)$ is greater than what is allowed by our models. We attribute the differences to calibration uncertainties in the atomic line ratios used by Parkin et al. (2013) and the simple geometry assumed by the PDR models.

(iv) We compare our two-component model for the centre region of M51 to similar models for the ULIRG Arp 220, the starburst galaxy M82 and the on-going merger NGC 4038/39. The characteristics of the cold component are comparable across all four systems, with the exception of the temperature of Arp 220, which is slightly higher. In the case of the warm component, the density in the centre region of M51 $\left(n\left(\mathrm{H}_{2}\right) \sim 10^{1.6}-10^{2.5}\right)$ is lower than the other three systems $\left(n\left(\mathrm{H}_{2}\right) \sim 10^{3}-10^{6.6}\right)$, while the temperatures are comparable. Interestingly, the warm gas mass fraction in M51 is higher than in the NGC 4038/9 merger and comparable to what is seen in Arp 220 and M82. We suggest that a more complete multiphase analysis of the molecular gas in M51 including both PDR models and mechanical heating would increase our understanding of this iconic system.

\section{ACKNOWLEDGEMENTS}

Herschel is an ESA space observatory with science instruments provided by European-led Principal Investigator consortia and with important participation from NASA. We thank the referee and the editor for constructive comments that improved the paper. This research was supported by grants from the Canadian Space Agency and the Natural Sciences and Engineering Research Council of Canada (PI: C. D. Wilson). PACS has been developed by a consortium of institutes led by MPE (Germany) and including UVIE (Austria); KU Leuven, CSL, IMEC (Belgium); CEA, LAM (France); MPIA (Germany); INAF-IFSI/OAA/OAP/OAT, LENS, SISSA (Italy); IAC (Spain). This development has been supported by the funding agencies BMVIT (Austria), ESA-PRODEX (Belgium), CEA/CNES (France), DLR (Germany), ASI/INAF (Italy) and CICYT/MCYT (Spain). SPIRE has been developed by a consortium of institutes led by Cardiff University (UK) and including Univ. Lethbridge (Canada); NAOC (China); CEA, LAM (France); IFSI, Univ. Padua (Italy); IAC (Spain); Stockholm Observatory (Sweden); Imperial College London, RAL, UCL-MSSL, UKATC, Univ. Sussex (UK) and Caltech, JPL, NHSC, Univ. Colorado (USA). This development has been supported by national funding agencies: CSA (Canada); NAOC (China); CEA, CNES, CNRS (France); ASI (Italy); MCINN (Spain); SNSB (Sweden); STFC (UK) and NASA (USA). HIPE is a joint development by the Herschel Science Ground Segment Consortium, consisting of ESA, the NASA Herschel Science Center and the HIFI, PACS and SPIRE consortia. This research has made use of the NASA/IPAC Extragalactic Database (NED) which is operated by the Jet Propulsion Laboratory, California Institute of Technology, under contract with the National Aeronautics and Space Administration. This research made use of the PYTHON plotting package MATPLOTLIB (Hunter 2007). This research made use of APLPY, an open-source plotting package for PYTHON hosted at http://aplpy.github.com. We would like to thank Mark Wolfire for providing the PDR model grids used in this paper. This work made use of HERACLES, 'The HERA CO-Line Extragalactic Survey (Leroy et al. 2009). This research was supported in part by the Grant-in-Aid for Scientific Research for the Japan Society of Promotion of Science (140500000638). IDL gratefully acknowledges the support of the Flemish Fund for Scientific Research (FWO Vlaanderen).

\section{REFERENCES}

Aniano G., Draine B. T., Gordon K. D., Sandstrom K., 2011, PASP, 123, 1218

Barcos-Muñoz L. et al., 2015, ApJ, 799, 10

Bendo G. J., Galliano F., Madden S. C., 2012, MNRAS, 423, 197

Blitz L., Magnani L., Mundy L., 1984, ApJ, 282, L9

Brunner G. et al., 2008, ApJ, 675, 316

Calzetti D. et al., 2010, ApJ, 714, 1256

Colombo D. et al., 2014a, ApJ, 784, 3

Colombo D. et al., 2014b, ApJ, 784, 4

Currie M. J., Draper P. W., Berry D. S., Jenness T., Cavanagh B., Economou F., 2008, in Argyle R. W., Bunclark P. S., Lewis J. R., eds, ASP Conf. Ser. Vol. 394, Astronomical Data Analysis Software and Systems XVII. Astron. Soc. Pac., San Francisco, p. 650

Dalcanton J. J. et al., 2009, ApJS, 183, 67

Dobbs C. L., Theis C., Pringle J. E., Bate M. R., 2010, MNRAS, 403, 625

Gaches B. A. L., Offner S. S. R., Rosolowsky E. W., Bisbas T. G., 2015, ApJ, 799, 235

Galametz M. et al., 2013, MNRAS, 431, 1956

Garcia-Burillo S., Guelin M., Cernicharo J., 1993, A\&A, 274, 123

Griffin M. J. et al., 2010, A\&A, 518, L3 
Hollenbach D., Kaufman M. J., Neufeld D., Wolfire M., Goicoechea J. R., 2012, ApJ, 754, 105

Hughes A. et al., 2013a, ApJ, 779, 44

Hughes A. et al., 2013b, ApJ, 779, 46

Hunter J. D., 2007, Comput. Sci. Eng., 9, 90

Ikeda M., Oka T., Tatematsu K., Sekimoto Y., Yamamoto S., 2002, ApJS, 139,467

Israel F. P., Tilanus R. P. J., Baas F., 2006, A\&A, 445, 907

Kamenetzky J. et al., 2012, ApJ, 753, 70

Kamenetzky J., Rangwala N., Glenn J., Maloney P. R., Conley A., 2014, ApJ, 795, 174

Kaufman M. J., Wolfire M. G., Hollenbach D. J., Luhman M. L., 1999, ApJ, 527,795

Kaufman M. J., Wolfire M. G., Hollenbach D. J., 2006, ApJ, 644, 283

Kazandjian M. V., Meijerink R., Pelupessy I., Israel F. P., Spaans M., 2012, A\&A, 542, A65

Kazandjian M. V., Meijerink R., Pelupessy I., Israel F. P., Spaans M., 2015, A\&A, 574, A127

Koda J. et al., 2009, ApJ, 700, L132

Koulouridis E., 2014, A\&A, 570, A72

Kramer C., Mookerjea B., Bayet E., Garcia-Burillo S., Gerin M., Israel F. P., Stutzki J., Wouterloot J. G. A., 2005, A\&A, 441, 961

Kramer C., Moreno R., Greve A., 2008, A\&A, 482, 359

Leroy A. K. et al., 2009, AJ, 137, 4670

Leroy A. K. et al., 2011, ApJ, 737, 12

Liszt H. S., Pety J., Tachihara K., 2009, A\&A, 499, 503

Maloney P. R., 1999, Ap\&SS, 266, 207

Meidt S. E. et al., 2013, ApJ, 779, 45

Mentuch Cooper E. et al., 2012, ApJ, 755, 165

Nakai N., Kuno N., Handa T., Sofue Y., 1994, PASJ, 46, 527

Naylor D. A. et al., 2010, Proc. SPIE, 7731, 773116

Nikola T., Geis N., Herrmann F., Madden S. C., Poglitsch A., Stacey G. J., Townes C. H., 2001, ApJ, 561, 203

Offner S. S. R., Bisbas T. G., Bell T. A., Viti S., 2014, MNRAS, 440, L81

Panuzzo P. et al., 2010, A\&A, 518, L37

Papadopoulos P. P., Thi W.-F., Viti S., 2004, MNRAS, 351, 147

Parkin T. J. et al., 2013, ApJ, 776, 65

Pety J., Lucas R., Liszt H. S., 2008, A\&A, 489, 217

Pety J. et al., 2013, ApJ, 779, 43

Pilbratt G. L. et al., 2010, A\&A, 518, L1

Plume R., Jaffe D. T., Tatematsu K., Evans N. J., II, Keene J., 1999, ApJ, 512,768

Poglitsch A. et al., 2010, A\&A, 518, L2

Rangwala N. et al., 2011, ApJ, 743, 94

Röllig M. et al., 2007, A\&A, 467, 187

Rose J. A., Searle L., 1982, ApJ, 253, 556

Roussel H. et al., 2007, ApJ, 669, 959

Satyapal S., Sambruna R. M., Dudik R. P., 2004, A\&A, 414, 825

Schinnerer E., Weiß A., Aalto S., Scoville N. Z., 2010, ApJ, 719, 1588

Schinnerer E. et al., 2013, ApJ, 779, 42

Schirm M. R. P. et al., 2014, ApJ, 781, 101

Schirm M. R. P., Wilson C. D., Madden S. C., Clements D. L., 2016, ApJ, 823,87

Schuster K. F., Kramer C., Hitschfeld M., Garcia-Burillo S., Mookerjea B., 2007, A\&A, 461, 143

Schweizer F. et al., 2008, AJ, 136, 1482

Scoville N., Young J. S., 1983, ApJ, 265, 148

Scoville N. Z., Yun M. S., Bryant P. M., 1997, ApJ, 484, 702

Shimajiri Y. et al., 2013, ApJ, 774, L20

Spinoglio L. et al., 2012, ApJ, 758, 108

Stanford S. A., Sargent A. I., Sanders D. B., Scoville N. Z., 1990, ApJ, 349, 492

Swinyard B. M. et al., 2014, MNRAS, 440, 3658

Tielens A. G. G. M., 2005, The Physics and Chemistry of the Interstellar Medium. Cambridge Univ. Press, Cambridge

Tielens A. G. G. M., Hollenbach D., 1985, ApJ, 291, 722

Tikhonov N. A., Galazutdinova O. A., Tikhonov E. N., 2009, Astron. Lett., 35,599
Tran H. D., 2001, ApJ, 554, L19

van der Tak F. F. S., Black J. H., Schöier F. L., Jansen D. J., van Dishoeck E. F., 2007, A\&A, 468, 627

Vlahakis C., van der Werf P., Israel F. P., Tilanus R. P. J., 2013, MNRAS, 433, 1837

Wilson C. D. et al., 2012, MNRAS, 424, 3050

Wolfire M. G., Hollenbach D., McKee C. F., 2010, ApJ, 716, 1191

Wu R. et al., 2013, A\&A, 556, A116

Wu R. et al., 2015, A\&A, 575, A88

Yun M. S., Ho P. T. P., Lo K. Y., 1993, ApJ, 411, L17

Zaritsky D., Rix H.-W., Rieke M., 1993, Nature, 364, 313

\section{APPENDIX A: SINGLE-COMPONENT FIT}

We fit a single component to the seven detected $\mathrm{CO}$ transitions $(\mathrm{J}=1-0$ to $\mathrm{J}=7-6)$, and both [ $\left.\mathrm{C}_{\mathrm{I}}\right]$ transitions, setting the $\mathrm{CO} \mathrm{J}=8-$ 7 transition as an upper limit, in the nucleus, centre and arm/interarm regions of M51, along with all three regions combined. We show the calculated fluxes from the 'best-fitting' solution compared to the measured fluxes in Fig. A1. We report the best-fitting solution for each of the physical parameters in Table A1, along with the $1 \sigma$ ranges for each parameter.

For all of the modelled regions, our single-component fits suggest that the $\mathrm{CO}$ emission is dominated by warm $(\sim 100-300 \mathrm{~K})$, relatively diffuse $\left(\lesssim 10^{2.4}-10^{2.8} \mathrm{~cm}^{-3}\right)$ molecular gas. While diffuse, $\mathrm{CO}$ emitting molecular gas has been observed within the Milky Way (e.g. see Pety, Lucas \& Liszt 2008; Liszt, Pety \& Tachihara 2009), most of the star-forming molecular gas is much colder than $200 \mathrm{~K}$. Furthermore, GMC scale observations of CO J $=1-0$ in M51 by Hughes et al. (2013a) find that the CO peak brightness temperature ranges from $T_{\mathrm{mb}}=1$ to $10 \mathrm{~K}$ on spatial scales of $\sim 50 \mathrm{pc}(\sim 1 \mathrm{arcsec})$. Assuming the $\mathrm{CO}$ emission fills the beam, the $\mathrm{CO}$ peak brightness temperature corresponds to the molecular gas

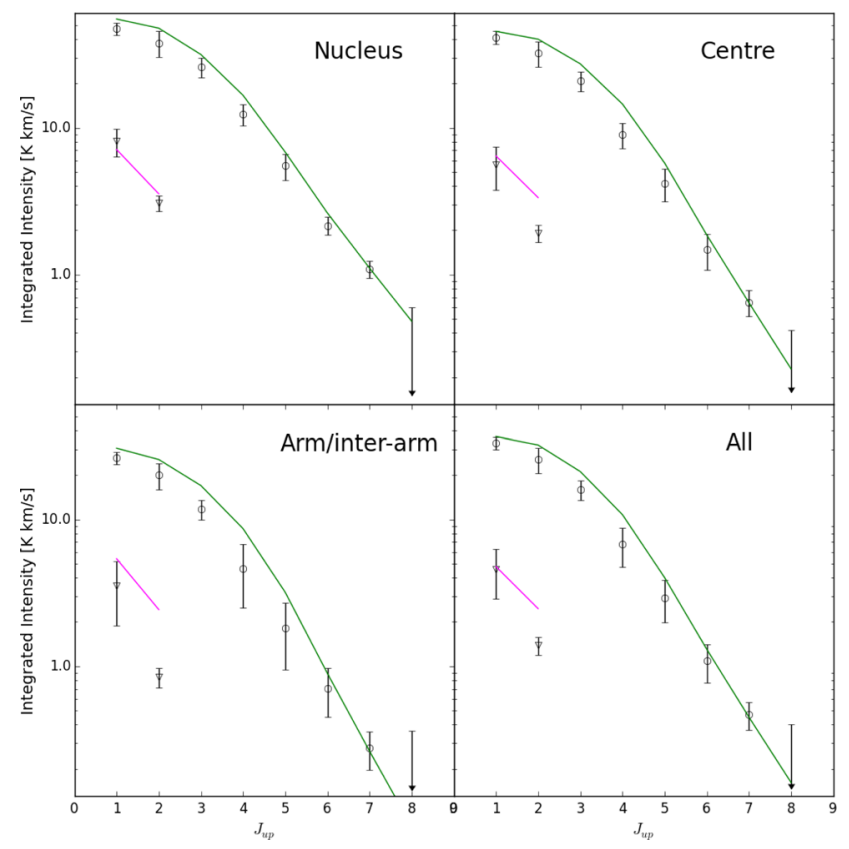

Figure A1. Measured and calculated spectral line energy distributions for the single-component fit for the nucleus (top-left), centre (top-right) and arm/interarm (bottom-left) regions, and for all the regions combined (bottom-right). The measured fluxes are shown by black circles $(\mathrm{CO})$ and triangles $([\mathrm{C} \mathrm{I}])$, while the calculated fluxes are shown by the green $(\mathrm{CO})$ and magenta ([C I]) solid lines. 
Table A1. Results from single-component non-LTE excitation analysis.

\begin{tabular}{|c|c|c|c|c|c|}
\hline \multicolumn{6}{|c|}{ Best fit ( $1 \sigma$ range) } \\
\hline Parameter & Nucleus & Centre & Arm/interarm & All & Units \\
\hline$T_{\text {kin }}$ & $234(162-320)$ & $141(112-256)$ & $104(90-236)$ & $141(110-277)$ & $\mathrm{K}$ \\
\hline $\log \left(N_{\mathrm{CO}}\right)$ & $18.68(17.85-18.64)$ & $18.70(17.69-18.67)$ & $18.74(17.05-18.53)$ & $18.56(17.36-18.57)$ & $\log \left(\mathrm{cm}^{-2}\right)$ \\
\hline $\log \left(\Phi_{\mathrm{A}}\right)$ & $-1.57(-1.72$ to -1.26$)$ & $-1.64(-1.74$ to -1.18$)$ & $-1.71(-1.76$ to -0.82$)$ & $-1.64(-1.74$ to -0.99$)$ & \\
\hline $\log \left(\left\langle N_{\mathrm{CO}}\right\rangle\right)$ & $16.92(16.57-17.02)$ & $17.00(16.49-17.01)$ & $16.31(16.21-16.83)$ & $16.51(16.35-16.91)$ & $\log \left(\mathrm{cm}^{-2}\right)$ \\
\hline
\end{tabular}
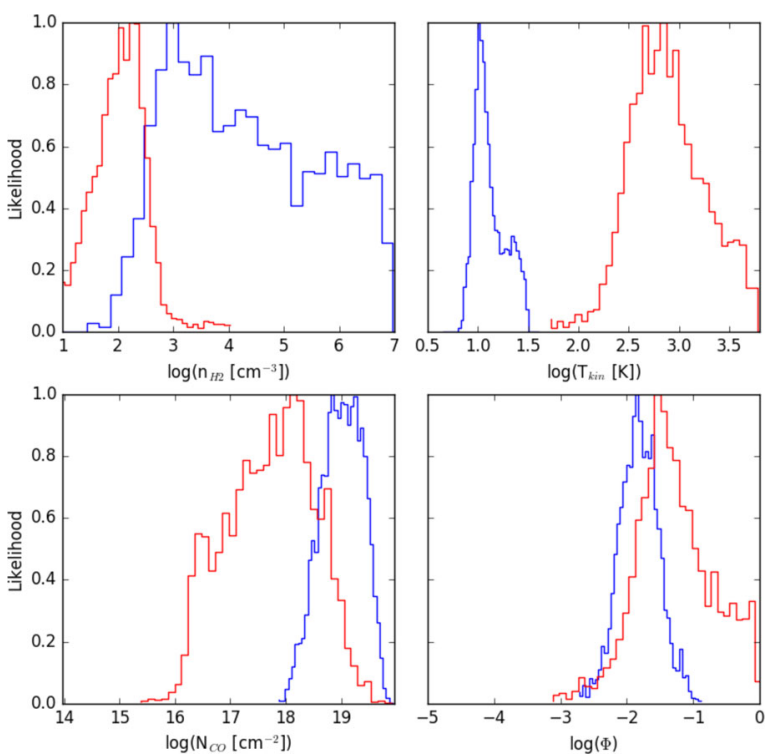

Figure B1. Probability distributions of four fitted parameters from the twocomponent RADEX model for the centre region of M51. The cold component distributions are shown in blue and the warm component distributions are shown in red for molecular gas density (top-left), kinetic temperature (topright), column density (bottom-left) and filling factor (bottom-right). temperature. If the molecular gas were at a temperature of $\sim 200 \mathrm{~K}$, as recovered in our single-component model, only $\sim 0.5-5$ per cent of the 1 arcsec beam would be filled. Given the typical sizes of GMCs $(10-100 \mathrm{pc})$, our single-component model does not appear likely to represent accurately the bulk of the molecular gas in M51.

\section{APPENDIX B: PARAMETER DISTRIBUTIONS}

Fig. B1 shows the probability distributions from the two-component Bayesian RADEX model fits to the centre region of M51. The distributions for the cold component fit are shown in blue while the distributions for the warm component fit are shown in red. Many of the distributions are decidedly asymmetric. In particular, the density distribution for the cold component fit is very broad (see also Table 4). The probability distributions for the fits to the nucleus and arm/interarm regions and to the average of all the data are very similar to the distributions for the centre region shown in Fig. B1.

This paper has been typeset from a $\mathrm{T}_{\mathrm{E}} \mathrm{X} / \mathrm{L} \mathrm{T} \mathrm{E} \mathrm{X}$ file prepared by the author. 